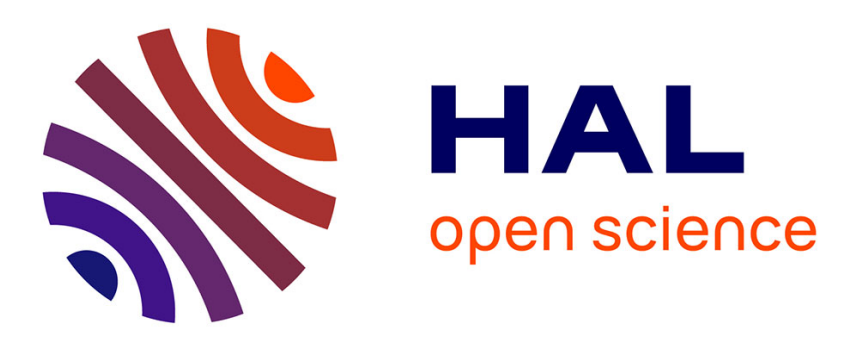

\title{
Sensitivity analysis of optimal transient growth for turbulent boundary layers
}

\author{
Frédéric Alizard, Jean-Christophe Robinet, Guillaume Filliard
}

\section{To cite this version:}

Frédéric Alizard, Jean-Christophe Robinet, Guillaume Filliard. Sensitivity analysis of optimal transient growth for turbulent boundary layers. European Journal of Mechanics - B/Fluids, 2015, 49, pp.373-386. 10.1016/j.euromechflu.2014.04.013 . hal-02569754

\section{HAL Id: hal-02569754 \\ https://hal.science/hal-02569754}

Submitted on 11 May 2020

HAL is a multi-disciplinary open access archive for the deposit and dissemination of scientific research documents, whether they are published or not. The documents may come from teaching and research institutions in France or abroad, or from public or private research centers.
L'archive ouverte pluridisciplinaire HAL, est destinée au dépôt et à la diffusion de documents scientifiques de niveau recherche, publiés ou non, émanant des établissements d'enseignement et de recherche français ou étrangers, des laboratoires publics ou privés. 


\title{
Sensitivity analysis of optimal transient growth for turbulent boundary layers
}

\author{
Frédéric Alizard ${ }^{\mathrm{a}, *}$, Jean-Christophe Robinet ${ }^{\mathrm{b}}$, Guillaume Filliard ${ }^{\mathrm{b}}$ \\ a Laboratoire DynFluid, Arts et Métiers ParisTech et CNAM, 151 Boulevard de l'Hôpital, 75013 Paris, France \\ b Laboratoire DynFluid, Arts et Métiers ParisTech, 151 Boulevard de l'Hôpital, 75013 Paris, France
}

Keywords:

Turbulent boundary layer

Optimal transient growth

Sensitivity to mean flow modification

\begin{abstract}
A B S T R A C T
Structural approaches based on modal decomposition of the flow dynamics have gained acceptance for a wide variety of turbulent shear flows. In this context, a singular value decomposition associated with a governing operator, aiming to model the linear amplification of coherent structures, is used to reproduce some fundamental motions in a turbulent boundary layer. In particular, as already found by Cossu et al. (2009), elongated streaky structures scaled in inner and outer units are identified. The sensitivity of these singular values to a mean flow modification is analysed. It is illustrated that the linear amplification of very large-scales which populate the outer motion is not affected when the leading singular value associated with the inner layer is damped. Moreover, we notice that the resulting optimal mean flow deviation is consistent with findings of Xu et al. (2007) in which the active control of a turbulent boundary layer is studied through direct numerical simulations.
\end{abstract}

\section{Introduction}

Since the pioneer work of Kline et al. [1] devoted to the understanding of the role of coherent structures in turbulent boundary layers (as streaks, vortices and hairpins), the structural point of view of wall turbulence gives new insight about turbulence dynamics (see the recent review of Jiménez [2]). The study of coherent structures, their spatial and time scales, their mutual interactions as well as their relationships, is fundamental to develop low-order models allowing us to describe mechanisms involved in wall turbulence (see Panton [3]). In that respect, as first suggested by Hamilton et al. [4], it is now commonly accepted that a self-sustaining process near the wall plays a major role in both the large-scale dynamics and the production of turbulent kinetic energy. The latter process relies on the formation of streamwise velocity streaks and their nonlinear breakdown. The linear part of such a process is caused by a so-called lift-up mechanism. Jiménez [5] also stressed that, in spite of the turbulence requires nonlinearity, there is strong evidence that the emergence of coherent structures as well as the main part of energy production mechanism in wall-bounded turbulence can be described by a linear model.

\footnotetext{
* Corresponding author.

E-mail address: frederic.alizard@cnam.fr (F. Alizard).
}

Structural approaches based on a linearization about a turbulent mean flow were first introduced by Reynolds and Hussain [6]. A linear dynamical system for the organized motion is specified where a triple decomposition of the instantaneous flow fields, which decorrelates the random part from the coherent part of the motion, is used. To model the coherent part of the Reynolds stress, the authors introduce an eddy viscosity by assuming that the largescales feel the dissipation from the smaller ones. However, by considering only asymptotic solutions, their analysis fails to describe accurately the coherent structures of a turbulent channel flow. In 2006, Del Alamo and Jiménez [7] reconsidered the linear model, proposed by Reynolds and Hussain [6], by tracking coherent structures which may be sustained in short times. The most amplified modes are computed for given time horizons and then compared with the turbulent structures in a channel flow. The analysis shows that the coherent structures which gain the maximum energy in the transient time reproduce the organization of turbulence in a channel flow both in terms of spatial and temporal scales. Such an attempt extends the previous results obtained by Butler and Farrell [8] in 1992 who used a similar transient growth theory (see Schmid and Henningson for a review in a laminar regime [9]) where the influence of the background turbulence onto the largescale motion is not included in the equations. To take into account the effect of the dissipation due to the turbulence, Butler and Farrell [8] constrain the time horizon with an appropriate turbulent eddy turnover time. Further investigations carried out by 
Pujals et al. [10] for a turbulent channel flow and Cossu et al. [11] for a turbulent boundary layer confirm the relevancy of linear optimal transient growth analyses, capable of providing characteristic features of wall-bounded flows. In particular, the previous authors emphasize that the modes are mostly amplified for infinitely elongated structures in the streamwise direction. In addition, the curve associated with the maximum energy gain exhibits two optima. For the turbulent boundary layer, Cossu et al. [11] show that an optimum in energy gain is scaled in inner units with a spanwise wavelength $\lambda_{z}^{+} \approx 81\left(\lambda_{z}^{+}=\lambda_{z} / \delta_{w}\right.$ with $\delta_{w}$ the wall viscous length scale). This spatial scale is consistent with the size of streaky structures involved in the autonomous cycle of near-wall turbulence [3]. A second optimum is scaled in outer units and is also seen to widely extend in the inner region. This observation is in agreement with experiments which report a scaling in outer units for the very large-scale motion lying in the outer region and a footprint of these structures in the inner layer [12]. Nevertheless, both the streamwise and spanwise wavelengths are overestimated by the most amplified structure whereas suboptimal modes are more consistent with experimental observations. More recently, Sharma and McKeon [13] described additional features in a turbulent channel flow by computing optimal response modes, i.e. modes leading to an optimal gain in energy with respect to a localized forcing. For instance, an appropriate superposition of the leading optimal modes predicts the organization of hairpin packets and the dynamics of very large-scale motion in the outer region, as described by Kim and Adrian [14]. Sharma and McKeon [13] illustrate that such very large-scale structures may arise naturally, rather than by a summation of hairpin packets. This theoretical analysis contradicts thus the model proposed by Kim and Adrian [14].

The singular value decomposition of a linear operator, the socalled propagator in the time domain and the resolvent in the frequency domain, is a common aspect of all the previous studies referenced above. Hence, the optimal modes associated with the most amplified singular values appear relevant to describe some fundamental features of coherent motions in turbulent shear flows. Nevertheless, such a linear analysis fails until now to predict some mutual dependency between the large-scale structures associated with the outer region and the coherent structures which populate the inner region. More particularly, as underlined by Mizuno and Jimenéz [15], the mutual interactions between the inner and outer layers for wall-bounded turbulence remain an open question. For example, how the large-scale motion in the outer region would continue to exist in the absence of the near-wall structures. Hwang and Cossu [16] have recently tackled this fundamental question through a Large Eddy Simulation of a turbulent channel flow with a Smagorinsky subgrid model. The authors show that an increase of the Smagorinsky constant is able to damp the smallscale coherent motion near the wall. As a consequence, the nearwall self-sustaining cycle is suppressed. The large-scale structures associated with the outer region are not modified. This analysis highlights that the large-scale dynamics in the outer region are almost independent of the near-wall cycle which dominates in the buffer layer. One may also remark that such an analysis further supports Townsend's wall similarity hypothesis [17] for rough walls stating that turbulence outside the inner layer is unaffected by surface condition. This hypothesis is quite controversial and is the scope of extensive research in both experiments [18-20] and numerical simulations [21-23]. Although Townsend's wall similarity hypothesis is still an open problem, there is some evidence that a part of discrepancies observed in the literature are caused by the different roughness geometries that are used [24].

From the above discussion, it seems relevant to assume that the linear optimal transient growth model used by Del Alamo and Jiménez [7], Pujals et al. [10] and Cossu et al. [11] is appropriate to describe both the inner layer and the outer layer coherent structures. Hence, in order to support the scenario proposed by Hwang and Cossu [16], one may investigate the amplification of optimal modes associated with the outer region when the most amplified singular value for the inner region is damped. It is interesting to further explore this fundamental question by means of a theoretical analysis based on the sensitivity of the leading singular values to mean flow modifications. A mean flow modification may be caused, for instance, by riblets, roughness elements or an actuator. In a linear stability framework, Bottaro et al. [25] seek to determine the effect on the asymptotic linear stability of a base flow deviation, of a given amplitude, from the ideal base flow. By introducing a so-called sensitivity function, a standard variational procedure is employed to target the optimal base-flow modification that maximizes (or minimizes) the temporal growth rate of a selected eigenmode, when assuming a fixed deviation parameter. A transition scenario involving the exponential growth of small disturbances is thus proposed by Bottaro et al. [25] in order to explain the triggering of turbulence for asymptotically linearly stable parallel shear flows as Couette flow. This concept is extended by Marquet et al. [26] in the control of the onset of unsteadiness behind a cylinder in a laminar regime. More recently, Meliga et al. [27] push forward such theoretical tools to control large-scale unsteadiness in a turbulent wake past a D-shaped cylinder. The latter authors compute the natural shedding mode by a linear stability analysis performed on the linearized U-RANS equations. Hence, Meliga et al. [27] assess sensitivity maps for the shedding mode with respect to small turbulent mean flow deviation caused by a small control cylinder. In particular, they identified the regions in which the small control cylinder has the most impact on both the frequency and the amplification rate of the shedding mode. All analyses discussed above focus on asymptotic behaviour. Brandt et al. [28] extend such a concept by considering the sensitivity of the optimal transient energy growth to a base flow modification, i.e. the leading singular value of the governing operator. The flat plate laminar boundary layer is used to illustrate their purpose. After having derived an analytical expression for the sensitivity function, the authors show the effect of a small base flow deviation on the lift-up mechanism and also its impact on the Tollmien-Schlichting waves. It is thus clear that sensitivity to mean flow modification analyses allows us to identify a transition scenario, mutual interactions between two competitive mechanisms, as well as passive control strategies.

The present research is an attempt to push forward the theoretical concepts of Brandt et al. [28] for turbulent flows. For that purpose, the sensitivity framework detailed in [28] is extended to the governing operator used to compute coherent structures in turbulent shear flows by $[7,10,11]$. More particularly, we would like to emphasize the mutual interaction between the inner and outer layer large-scale motions and also to provide a theoretical framework to damp the autonomous cycle of near-wall turbulence. To achieve such a goal, the line of thought is as follows: after having presented the turbulent boundary layer mean flow profiles, we address the basic concepts of optimal transient growth and sensitivity analysis for a turbulent mean flow. Then, we present the sensitivity functions for a mean flow modification for both the optimal modes associated with the inner and outer regions. The optimal mean flow deviation which stabilizes the optimal mode for the inner region is thus tackled. Hence, the mutual interactions between the inner and outer large-scale motions are highlighted, as well as the optimal distorted turbulent mean flow. A final part is dedicated to some discussions and prospects.

\section{Mean flow and eddy viscosity}

The explicit expression for the turbulent mean velocity given by Monkewitz et al. [29] is used

$$
U^{+}=U_{i}^{+}\left(y^{+}\right)-U_{\log }^{+}\left(y^{+}\right)+U_{e}^{+}\left(R e_{\delta^{*}}\right)-U_{w}^{+}(\eta),
$$$$
\text { with } U^{+}=U / u_{\tau}
$$ 

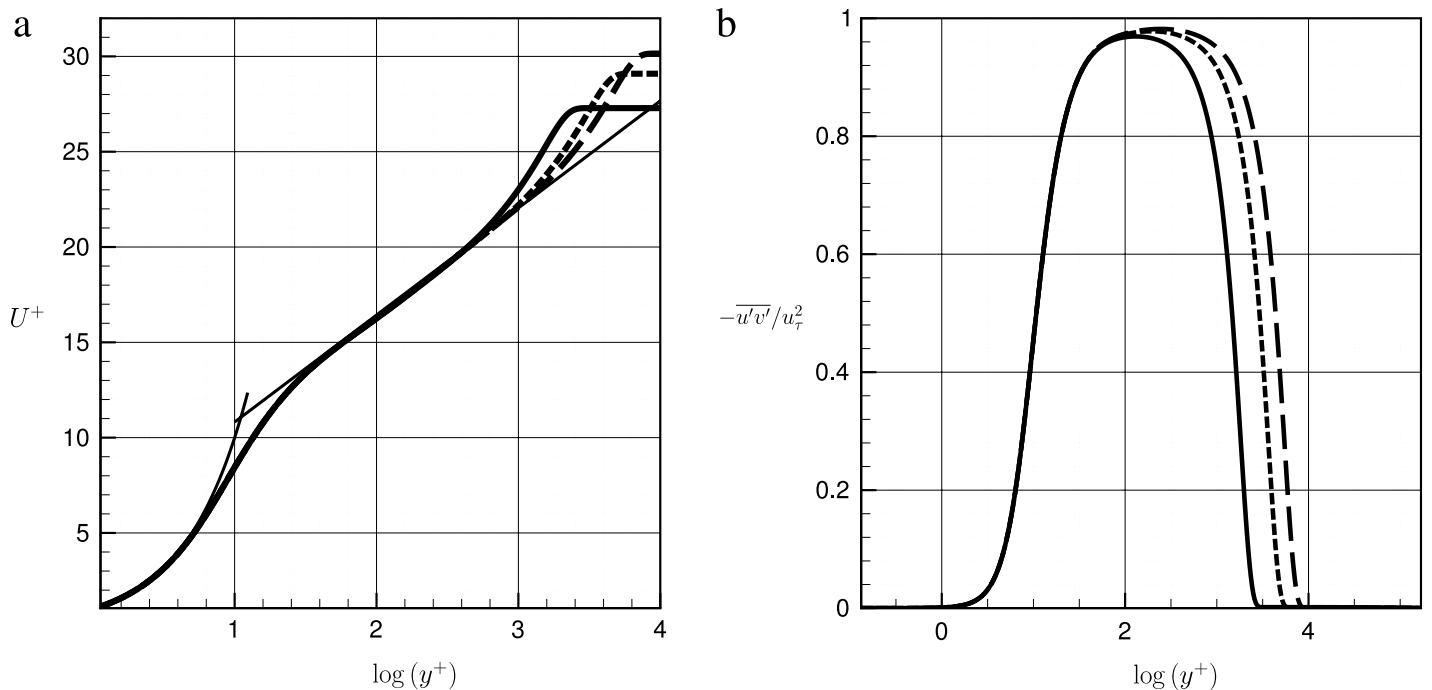

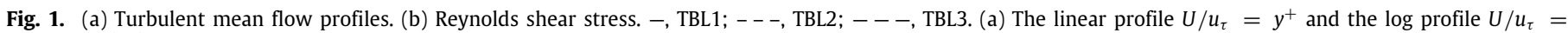
$1 / 0.41 \log \left(y^{+}\right)+5.2$ are also represented in solid lines.

where $U_{i}^{+}, U_{\log }^{+}$and $U_{w}^{+}$represent the inner layer velocity, a log layer velocity and a wake function respectively, and $U_{e}$ is the freestream velocity. The velocity components are nondimensionalized by the friction velocity $u_{\tau}=\sqrt{\tau_{w} / \rho}$, where $\tau_{w}$ and $\rho$ are respectively the wall shear stress and the mean density; $y^{+}$is the wall-normal coordinate scaled in inner units $\left(y^{+}=y / \delta_{w}\right.$, where $v$ is the mean kinematic viscosity and $\delta_{w}=v / u_{\tau}$ is the wall viscous length scale), $\eta$ is the wall-normal coordinate scaled in outer units $(\eta=y / \Delta$, where $\Delta$ is the Rotta-Clauser outer length scale), and $\operatorname{Re}_{\delta^{*}}$ is the Reynolds number based on the boundary layer displacement thickness. Finally, we note $\delta_{99}$ the boundary layer thickness corresponding to the normal distance to the wall where $U$ reaches $0.99 U_{e}$.

As noticed by Reynolds and Hussain [6], the dynamical equations governing the linear dynamics of organized waves in turbulent flows have to include a term representing the oscillation of the background Reynolds stress. A simple model based on an eddy viscosity, noted $v_{t}$ hereafter, is then proposed by the previous authors. For that purpose, under a parallel flow assumption, the eddy viscosity is defined as

$$
-\overline{u^{\prime} v^{\prime}}=\frac{v_{t}}{R e} \frac{d U}{d y}
$$

where the Reynolds shear stress $-\overline{u^{\prime} v^{\prime}}$ associated with the mean flow $U$ is retrieved by integrating the mean streamwise momentum equation in the wall-normal direction. The eddy viscosity is thus computed by using Eq. (2) inside the boundary layer. An extrapolation of the eddy viscosity in the potential region is then performed because $v_{t}(y)$ is numerically undefined outside the boundary layer (for more details see Cossu et al. [11]).

In the next, three Reynolds numbers are considered and referenced in Table 1 . The mean velocity profiles $U$ as well as the Reynolds stress component $-\overline{u^{\prime} v^{\prime}}$ are shown in Fig. 1.

\section{Transient growth for turbulent flows}

\subsection{Some theory}

The instantaneous flow field is decomposed into three contributions: a mean flow, a coherent part of the motion and random fluctuations. By introducing this triple decomposition into the
Table 1

Turbulent boundary layers' properties. $C_{f}=2 \tau_{w} /\left(\rho U_{e}^{2}\right) ; R e_{\tau}=u_{\tau} \delta_{99} / \nu=\delta_{99} / \delta_{w}$, $\operatorname{Re}_{\theta}=U_{e} \theta / v$ with $\theta$ the momentum thickness.

\begin{tabular}{llll}
\hline Dataset & $R e_{\tau}$ & $R e_{\theta}$ & $C_{f}\left(\times 10^{3}\right)$ \\
\hline TBL1 & 2213 & 7381 & 2.68 \\
TBL2 & 4464 & 15105 & 2.36 \\
TBL3 & 6636 & 22923 & 2.20 \\
\hline
\end{tabular}

Navier-Stokes equations and subtracting the time averaging from the phase averaging, the governing equations associated with the linear dynamics for the large-scale structures are, written in a dimensionless form

$$
\begin{aligned}
\frac{\partial \mathbf{u}}{\partial t}= & -(\mathbf{U} \cdot \nabla) \mathbf{u}-(\mathbf{u} \cdot \nabla) \mathbf{U}-\nabla(p) \\
& +\nabla \cdot\left(\frac{1+v_{t}}{\operatorname{Re}}\left(\nabla \mathbf{u}+\nabla^{t} \mathbf{u}\right)\right)
\end{aligned}
$$

$\nabla \cdot \mathbf{u}=0$

where an eddy viscosity model is used to relate the oscillation of the background Reynolds stress to the strain rate oscillation, as suggested by Reynolds and Hussain [6]. Moreover, $\mathbf{u}=(u, v, w)^{t}$ are the velocity components of the large-scale structures, $\mathbf{U}$ is the mean flow, $p$ is the pressure component and $R e$ is the Reynolds number. The system (3) is initialized with $\mathbf{u}(\mathbf{x}, t=0)=\mathbf{u}_{0}(\mathbf{x})$. In the next, we assume that the mean flow is parallel along the streamwise direction, i.e. $\mathbf{U}=(U(y), 0,0)^{t}$. We use a Fourier decomposition for the streamwise and spanwise directions:

$\mathbf{u}=\hat{\mathbf{u}}(y, t) e^{i(\alpha x+\beta z)}$

where $y$ denotes the wall-normal direction and $\alpha, \beta$ the wavenumbers associated with $x$ and $z$, respectively. The streamwise and spanwise wavelengths are denoted as $\lambda_{x}=2 \pi / \alpha$ and $\lambda_{z}=2 \pi / \beta$, respectively. The pressure term in (3) is rewritten as a function of velocity fields

$\hat{p}=\mathcal{K} \hat{\mathbf{u}}$.

The system (3) is thus expressed as

$$
\left(\frac{\partial}{\partial t}-\mathcal{A}\right) \hat{\mathbf{u}}=0, \quad \hat{\mathbf{u}}(0)=\hat{\mathbf{u}}_{0}
$$


where the operator $\mathcal{A}$ is

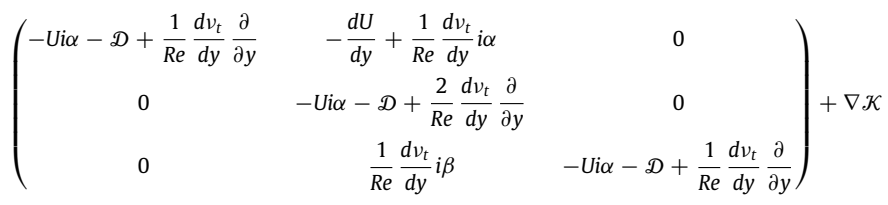

with $\mathscr{D}=\left(1+v_{t}\right) / \operatorname{Re}\left(\alpha^{2}+\beta^{2}-\partial^{2} / \partial y^{2}\right)$. To investigate the space-time organization of coherent structures evolving in a turbulent mean flow, it is interesting to compute modes which are mostly amplified during short time (see for instance $[7,11]$ ). Therefore, the amplification of the energy associated with coherent structures is measured by using a kinetic energy norm based on the inner product $\langle$,

$\|\mathbf{u}(t)\|^{2}=\langle\mathbf{u}(t), \mathbf{u}(t)\rangle=\int_{\mathcal{V}} \mathbf{u}^{h} \mathbf{u} d \mathcal{V}$

where $\bullet^{h}$ denotes the transconjugate. The adjoint system is derived using an inner product in the time-space domain $[0, T] \times \mathcal{V}$

$$
\left(-\frac{\partial}{\partial t}-\mathcal{A}^{+}\right) \hat{\mathbf{u}}^{+}=0, \quad \hat{\mathbf{u}}^{+}(0)=\hat{\mathbf{u}}_{0}^{+}
$$

with $\mathscr{H}$ the counterpart of $\mathcal{K}$ for the adjoint pressure and $\mathscr{A}^{+}$the adjoint operator of $\mathcal{A}$ with respect to $\langle$,$\rangle (its derivation is detailed$ in the Appendix)

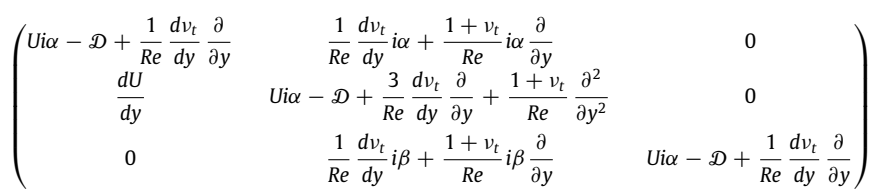

$-\nabla \mathscr{H}$.

The mode leading to a maximum growth in kinetic energy at time $T$ is associated with the largest singular value of $\exp (\mathcal{A} T)$ :

$\exp \left(\mathcal{A}^{+} T\right) \exp (\mathscr{A} T) \hat{\mathbf{u}}(0)=\sigma^{2} \hat{\mathbf{u}}(0)$

where the optimal gain in energy is given by $\sigma^{2}[9]$.

\subsection{Numerical methods}

The direct and adjoint operators, $\mathcal{A}$ and $\mathcal{A}^{+}$respectively, are discretized by using a Chebyshev collocation method in the normal direction $y$ for the divergence-free velocity components. The velocity components are set equal to zero at the wall and are assumed to vanish in the freestream. The singular value decomposition of the discrete exponential operator exp $(\mathcal{A} T)$ is carried out through the eigenvalues problem (5). For that purpose, the library EXPOKIT [30] is used to compute the matrix exponential for both the direct and adjoint operators and the $L A P A C K$ library is employed to compute the eigenvalues and corresponding eigenvectors. Accurate results are obtained for $n_{y}=300$ with $n_{y}$ the number of collocation points.

\section{Sensitivity to mean flow modifications}

\subsection{Sensitivity functions}

To study the sensitivity of any singular values $\sigma^{2}$ to any mean flow variation $\delta U$, we introduce the sensitivity function $\nabla_{U} \sigma^{2}$ as follows:

$\delta \sigma^{2}=\left\langle\nabla_{U} \sigma^{2}, \delta U\right\rangle$

where $\delta \sigma^{2}$ denotes the deviation of the singular value $\sigma^{2}$. The sensitivity function $\nabla_{U} \sigma^{2}$ is expressed by using the method of Lagrangian multipliers as exposed by Brandt et al. [28] where further details can be found. The objective function is then the singular value $\sigma^{2}$, the control is $U$ and the additional constraints are given by the system (5) which is enforced through Lagrange multipliers referenced hereafter as $\mathbf{a}^{+}, \mathbf{b}^{+}$and $\mathbf{f}^{+}$. Hence, we define the Lagrangian function $\mathcal{F}$

$$
\begin{aligned}
\mathcal{F} & \left(\mathbf{a}, \mathbf{b}, \mathbf{f}, \mathbf{a}^{+}, \mathbf{b}^{+}, \mathbf{f}^{+}, \sigma^{2}, U\right) \\
= & \sigma^{2}-\left\langle\mathbf{a}^{+}, \exp (\mathcal{A} T) \mathbf{f}-\mathbf{a}\right\rangle-\left\langle\mathbf{b}^{+}, \exp \left(\mathcal{A}^{+} T\right) \mathbf{a}-\mathbf{b}\right\rangle \\
& -\left\langle\mathbf{f}^{+}, \sigma^{2} \mathbf{f}-\mathbf{b}\right\rangle
\end{aligned}
$$

with $\mathbf{a}=\left(a_{1}, a_{2}, a_{3}\right)^{t}, \mathbf{b}=\left(b_{1}, b_{2}, b_{3}\right)^{t}$ and $\mathbf{f}=(f, g, h)^{t}$ (similarly for $\bullet^{+}$). By using a combination of the different constraints, it is clear that $\sigma^{2}$ is a singular value of $\exp (\mathscr{A} T)$, where the right singular vector is the initial condition: $\mathbf{f}=\hat{\mathbf{u}}_{0}$. A common way to derive an analytical expression of $\nabla_{U} \sigma^{2}$ is to perform an analogy in the frequency domain [28], since we need to differentiate a time-dependent operator in the time domain. We consider the asymptotic response to a harmonic forcing with a spatial shape given by $\mathbf{f}$ and a circular frequency $\omega$. We assume that all eigenvalues of the operator $\mathcal{A}$ are damped temporally, which is consistent with the previous results of Cossu et al. [11] for a turbulent boundary layer and Del Alamo and Jiménez [7] for a turbulent channel flow. In this context, the long-time behaviour of the coherent structure is driven by the resolvent operator, $\mathcal{R}$, through

$\mathbf{a}=\mathcal{R} \mathbf{f}=\frac{\mathbf{f}}{i \omega \mathbf{I}-\mathcal{A}}$.

Note that this operator is an equivalent to the time propagator $\exp (\mathcal{A} T)$ in the frequency domain. Therefore, the Lagrangian function in the frequency domain is derived by replacing the time derivative with $-i \omega$ in (7)

$$
\begin{aligned}
\mathscr{F} & \left(\mathbf{a}, \mathbf{b}, \mathbf{f}, \mathbf{a}^{+}, \mathbf{b}^{+}, \mathbf{f}^{+}, \sigma^{2}, U\right) \\
= & \sigma^{2}-\left\langle\mathbf{a}^{+},(i \omega \mathbf{I}-\mathcal{A}) \mathbf{a}-\mathbf{f}\right\rangle-\left\langle\mathbf{b}^{+},\left(-i \omega \mathbf{I}-\mathcal{A}^{+}\right) \mathbf{b}-\mathbf{a}\right\rangle \\
& -\left\langle\mathbf{f}^{+}, \sigma^{2} \mathbf{f}-\mathbf{b}\right\rangle .
\end{aligned}
$$

In this case, the forcing term $\mathbf{f}$ is an eigenvector of the operator $\mathcal{R}^{+} \mathcal{R}$, such as

$$
\left(-i \omega \mathbf{I}-\mathcal{A}^{+}\right)^{-1}(i \omega \mathbf{I}-\mathcal{A})^{-1} \mathbf{f}=\sigma^{2} \mathbf{f} .
$$

The total variation of $\mathcal{F}$ is

$$
\begin{aligned}
\delta \mathcal{F}= & \left\langle\frac{\partial \mathcal{F}}{\partial \mathbf{a}}, \delta \mathbf{a}\right\rangle+\left\langle\frac{\partial \mathcal{F}}{\partial \mathbf{b}}, \delta \mathbf{b}\right\rangle+\left\langle\frac{\partial \mathcal{F}}{\partial \mathbf{f}}, \delta \mathbf{f}\right\rangle+\left\langle\frac{\partial \mathcal{F}}{\partial \mathbf{a}^{+}}, \delta \mathbf{a}^{+}\right\rangle \\
& +\left\langle\frac{\partial \mathcal{F}}{\partial \mathbf{b}^{+}}, \delta \mathbf{b}^{+}\right\rangle+\left\langle\frac{\partial \mathcal{F}}{\partial \mathbf{f}^{+}}, \delta \mathbf{f}^{+}\right\rangle+\left\langle\frac{\partial \mathcal{F}}{\partial \sigma^{2}}, \delta \sigma^{2}\right\rangle \\
& +\left\langle\frac{\partial \mathcal{F}}{\partial U}, \delta U\right\rangle .
\end{aligned}
$$

The condition of optimality is reached when (10) is equal to zero. This is fulfilled when all terms in (10) vanish to zero. Imposing the stationarity of $\mathcal{F}$ with respect to the Lagrange multipliers, $\mathbf{a}^{+}, \mathbf{b}^{+}$ and $\mathbf{f}^{+}$, yields the state equation (9). The variation with respect to b gives the direct equation

$(i \omega \mathbf{I}-\mathcal{A}) \mathbf{b}^{+}=\mathbf{f}^{+}$.

Similarly, the variation associated with a leads to the adjoint system

$\left(-i \omega \mathbf{I}-\mathcal{A}^{+}\right) \mathbf{a}^{+}=\mathbf{b}^{+}$.

The variation with respect to $\mathbf{f}$ leads to the following condition:

$\sigma^{2} \mathbf{f}=\mathbf{a}^{+}$. 

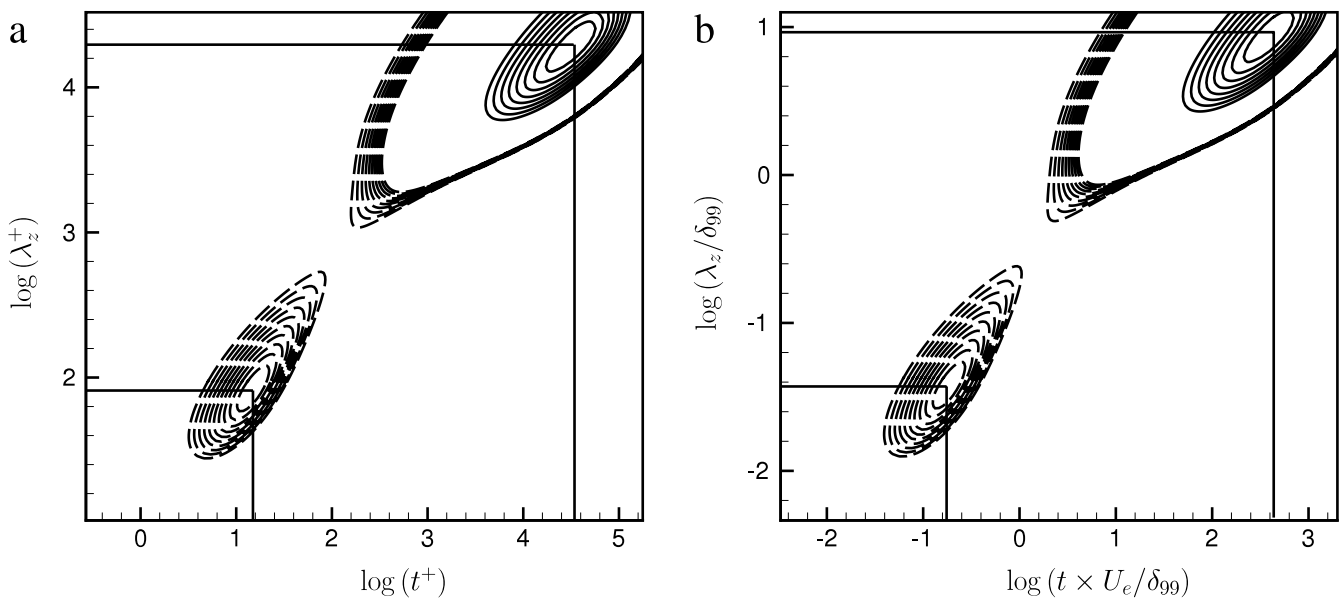

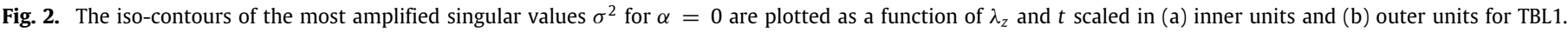
- contours levels range from 30 to 65 . - - - contours levels range from 1.8 to 2.7.

Then, the variation associated with $\sigma^{2}$ yields the normalization criterion

$\left\langle\mathbf{f}^{+}, \mathbf{f}\right\rangle=1$.

By using Eqs. (11)-(13), we obtain

$\left(-i \omega-\mathcal{A}^{+}\right)^{-1}(i \omega-\mathcal{A})^{-1} \mathbf{f}^{+}=\sigma^{2} \mathbf{f}^{+}$.

Hence, $\mathbf{f}^{+}$and $\mathbf{f}$ are collinear. The normalization condition (14) gives thus the following relationships:

$$
\left\{\begin{array}{l}
\mathbf{f}^{+}=\mathbf{f} \\
\mathbf{a}^{+}=\mathbf{b} \\
\mathbf{b}^{+}=\mathbf{a} .
\end{array}\right.
$$

Finally, the variation with respect to the control $U$ gives an expression of the sensitivity function for mean flow deviation

$$
\begin{aligned}
& \nabla_{U} \sigma^{2} \\
& \quad=2 \sigma^{2} \Re\left(f^{*} i \alpha a_{1}+g^{*} i \alpha a_{2}+h^{*} i \alpha a_{3}-a_{2} \frac{d f^{*}}{d y}-f^{*} \frac{d a_{2}}{d y}\right)
\end{aligned}
$$

where $\bullet$ * denotes the complex conjugate and $\Re$ the real part. One may notice that $\nabla_{U} \sigma^{2}$ is only governed by the input (f) and output (a) of our system. By considering that the same system is studied in the time domain, the sensitivity function associated with the Lagrangian (7) is equivalent to (16) where $\mathbf{f}$ is the initial condition and $\mathbf{a}$ is related to $\mathbf{f}$ which is propagated forward in time $T$.

\subsection{Optimal mean flow deviation}

The results obtained from the previous section can be used to determine the optimal mean flow deviation, i.e. that yields the largest change in the singular value selected, for a mean flow modification of a given magnitude (see Bottaro et al. [25]). The finding of such a deviation is of great interest because a passive/active control strategy may greatly benefit from damping the amplification of the largest singular values. Hence, we introduce the deviation parameter, $r$, as

$r^{2}=\int\left(U-U_{\text {ref }}\right)^{2} d y$

with $U_{\text {ref }}$ the mean flow without any modifications. Let us consider the Lagrangian function $\mathcal{L}$

$\mathcal{L}\left(U, \sigma^{2}, r, \gamma\right)=\sigma^{2}-\gamma\left(\int\left(U-U_{\mathrm{ref}}\right)^{2} d y-r^{2}\right)$ where $\gamma$ is a Lagrange multiplier which enforces the constraint (17). By using the sensitivity function $\nabla_{U} \sigma^{2}$, a variational approach to minimize $\sigma^{2}$ yields (see [25])

$$
\left\{\begin{array}{l}
U=U_{\mathrm{ref}}+\frac{1}{2 \gamma} \nabla_{U} \sigma^{2} \\
\text { with } \gamma=-\sqrt{\frac{1}{4 r^{2}} \int\left(\nabla_{U} \sigma^{2}\right)^{2} d y .}
\end{array}\right.
$$

The previous system is solved iteratively to determine the optimal mean flow modification for a given deviation parameter.

\section{Sensitivity analysis of incompressible turbulent boundary layers}

\subsection{Transient growth and coherent structures}

The objective of the present work is to understand the influence of a small deviation of the turbulent mean flow on the optimal energy growth. For that purpose, we report the optimal energy growth which is only considered for validation. Our results confirm that the maximum energy growth is reached for structures that are infinitely elongated along the streamwise direction, i.e. corresponding to $\alpha=0$. In particular, the optimal transient growth exhibits a double peak as illustrated in Fig. 2. A primary peak, referenced hereafter as the inner peak, is associated with the inner layer and its characteristic features are scaled in inner units $\left(\delta_{w}\right.$ and $\delta_{w} / u_{\tau}$ for the spatial and time scales respectively). A secondary peak, referenced hereafter as the outer peak, is observed in the outer region and the underlying coherent structures are scaled in outer units ( $\delta_{99}$ and $\delta_{99} / U_{e}$ ). As one may observe in Fig. 2 , the inner peak is reached for $\lambda_{z}^{+}=(2 \pi / \beta) / \delta_{w} \approx 81$ with a maximum gain in energy $\sigma^{2}=2.81$ (independent of the Reynolds number) while the outer peak is reached for $\lambda_{z} / \delta_{99}=(2 \pi / \beta) / \delta_{99} \approx 8$ with a maximum gain in energy $\sigma^{2}$ varying from 70 to 83 . For the sake of conciseness, only the case TBL1 is displayed in Fig. 2 but similar results are provided by TBL2 and TBL3. The optimal modes at the initial and optimal time $\left(t_{\mathrm{opt}}\right)$, i.e. the time necessary to reach the optimal gain in energy, are shown in Fig. 3. A so-called lift-up effect is seen to be the mechanism responsible for the growth in energy for both the inner and outer peaks. When compared with optimal modes obtained by Cossu et al. [11] for similar turbulent boundary layers, our results are quite in agreement both in terms of spanwise extends and amplifications in kinetic energy. To evaluate the robustness of the results discussed hereafter, the effect of the 

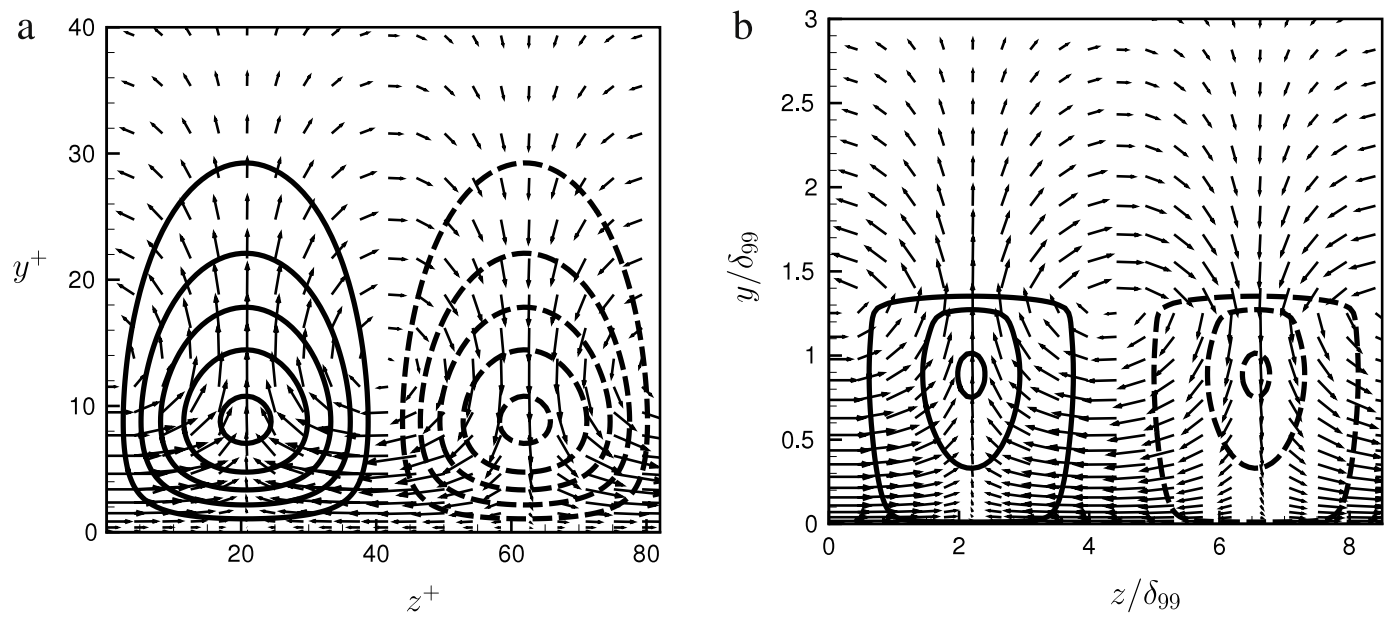

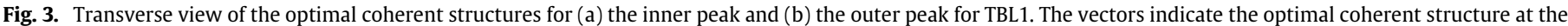
initial time in the cross-section, and the contours represent the streamwise component of the optimal coherent structure at the time $t=t_{\mathrm{opt}}$.

eddy viscosity on the outer peak is further examined by assuming $v_{t}(y)=v_{t \max }$ where $v_{t \max }=\max _{y} v_{t}(y)$. In particular, we found that the optimal growth is reduced $(\approx 5.64$ for TBL 1$)$ and is reached for infinitely elongated structures along the streamwise direction and $\lambda_{z} \approx 4.1 \delta_{99}$. These findings are also in agreement with values found by Cossu et al. [11].

However, as stressed by the latter authors, the inner layer dynamics is well represented by the optimal mode (i.e. for $\alpha=0$ ) in terms of spanwise wavelength (of the same order as $100 \delta_{w}$ ) whereas the very large-scale structures populating the outer region have a finite size in the streamwise direction (see Tomkins and Adrian [31]). In particular, by using two-point correlations of the streamwise velocity fluctuations, the recent experiments of Hutchins and Marusic [12] show that characteristic spanwise and streamwise scales may extend up to $0.25 \delta_{99}$ and $2 \delta_{99}$ respectively. This has for consequence to incline the structures along the spanwise direction. In addition, it is now well accepted that such very large-scale structures are inclined upward from the wall at approximatively $12^{\circ}-15^{\circ}$ [32]. Hence, it is also interesting to consider the optimal modes associated with the outer region for several $\alpha$ and $\beta$. We introduce two parameters which characterize the structure inclination angles in the planes $(x, y)$ and $(x, z)$ as

$\theta_{x y}=\operatorname{Atan}\left(\frac{y_{\max }}{\lambda_{x}}\right)$, and $\theta_{x z}=\operatorname{Atan}\left(\frac{\lambda_{z}}{\lambda_{x}}\right)$

with $y_{\max }$ the wall-normal position where the streamwise component of the optimal mode reaches a maximum. In the next, we consider the maximum of $\sigma^{2}$ over $t, \max _{(t)} \sigma^{2}$, as well as the maximum of $\sigma^{2}$ over both $\beta$ and $t, \max _{(t, \beta)} \sigma^{2}$, for several values of $\alpha$. In Fig. 4(a), the distribution of $\max _{t} \sigma^{2}$ is reported for four values of $\lambda_{x}=2 \pi / \alpha$. It may be observed that the maximum gain is decreasing with decreasing $\lambda_{x}$. In addition, the spanwise extend is seen to increase with $\lambda_{x}$. In Fig. 4(b), we plot the distribution of the spanwise wavelength $\lambda_{z} / \delta_{99}\left(\theta_{x y}\right)$ in the neighbourhood of $\max _{(\beta, t)} \sigma^{2}$ for the same values of $\lambda_{x}$. In Fig. 4(c), the distribution of the spanwise wavelength $\lambda_{z} / \delta_{99}$ is depicted as a function of $\theta_{x z}$, for the same parameters. The case TBL1 is used to illustrate our purpose. It is clear that a large variety of structures populating the outer region may be amplified. In particular, the coherent structure having an inclination angle upward from the wall $\approx 13^{\circ}$ has a characteristic spanwise wavelength $\lambda_{z} / \delta_{99} \approx 2$ which is more consistent with the experimental observations. Finally, cross-sections of the streamwise component for the optimal mode associated with $\lambda_{z} / \delta_{99} \approx 2$ and $\theta_{x y}=12.7$ are plotted in Fig. 5 . One may also notice that the structure inclination angle $\theta_{x y}$ is found to be invariant over a large range of Reynolds numbers, which is in agreement with the experiments of Marusic and Heuer [32].

\subsection{Sensitivity functions for coherent structures in the inner and outer regions}

The sensitivity functions $\nabla_{U} \sigma^{2}$ are computed using Eq. (16). An optimal mode is thus selected for a given couple $(\alpha, \beta)$. The initial condition and its propagation forward in time (equal to $t_{\mathrm{opt}}$ ) are then computed. The sensitivity functions for the inner peak are rewritten by using a scaling in inner units. In this context, let us consider a small singular value deviation written in inner variables

$$
\begin{aligned}
\delta \sigma^{2} & =\left(\nabla_{U} \sigma^{2}, \delta U\right) \\
& =\int_{y} \nabla_{U} \sigma^{2}(y) \delta U d y=\int_{y^{+}} \nabla_{U} \sigma^{2}(y) \delta U \delta_{w}^{2} d y^{+} .
\end{aligned}
$$

In a similar way, $\delta \sigma^{2}$ is expressed in outer variables for the outer motion

$\delta \sigma^{2}=\int_{y / \delta_{99}} \nabla_{U} \sigma^{2}(y) \delta U \delta_{99}^{2} d\left(y / \delta_{99}\right)$.

In that respect, the sensitivity functions scaled in inner and outer units, referenced as $\left(\nabla_{U} \sigma^{2}\right)^{\text {in }}$ and $\left(\nabla_{U} \sigma^{2}\right)^{\text {out }}$ respectively, are

$$
\left\{\begin{array}{l}
\left(\nabla_{U} \sigma^{2}\right)^{\text {in }}=\nabla_{U} \sigma^{2}(y) \delta_{w}^{2} \\
\left(\nabla_{U} \sigma^{2}\right)^{\text {out }}=\nabla_{U} \sigma^{2}(y) \delta_{99}^{2}
\end{array}\right.
$$

In Fig. 6, we show the sensitivity functions $\left(\nabla_{U} \sigma^{2}\right)^{\text {in }}$ and $\left(\nabla_{U} \sigma^{2}\right)^{\text {out }}$ for the optimal coherent structures infinitely elongated in the streamwise direction with respect to the inner (Fig. 6(a)) and outer peaks (Fig. 6(b)). The sensitivity functions displayed in Fig. 6(a) and (b) exhibit a similar shape. One may remark that the distribution of the sensitivity function along the wall-normal position is related to the most efficient minimal mean flow deviation aiming to modify the leading singular value. In particular, for the inner peak, this mean flow deviation is associated with a reduction of the mean velocity at the wall and an increase of the mean velocity in the overlap region between the inner and the outer layers. Such a mean flow modification could be induced by roughness elements [23], riblets [33] or constant near-wall forcing [34] for instance. It is also interesting to remark that a similar sensitivity function has been recently observed by Brandt et al. [28] who have considered the amplification of streaky structures in a laminar regime. When focusing on a sensitivity function associated with the outer region, 

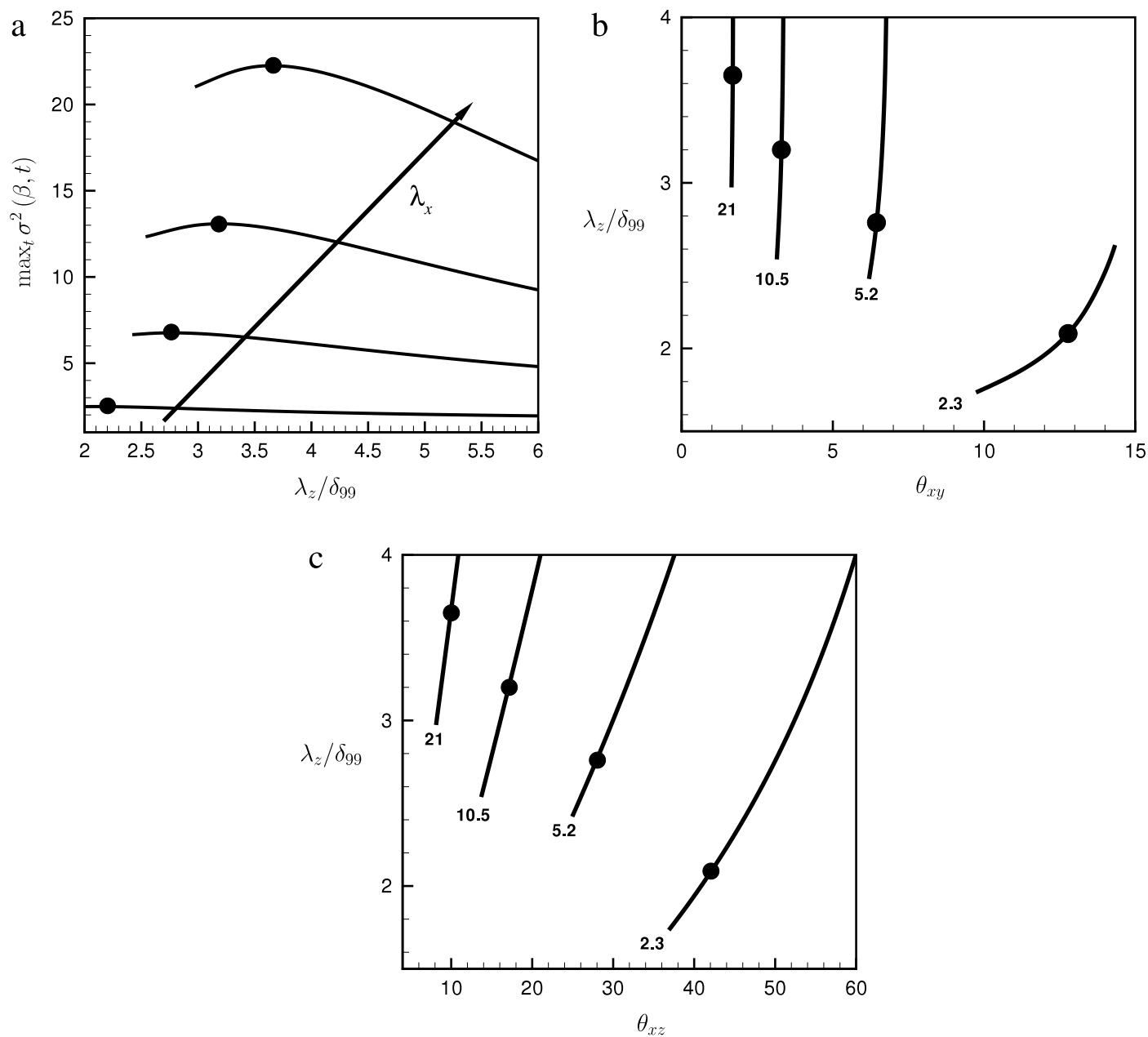

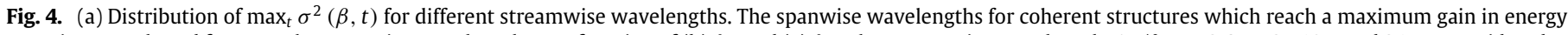

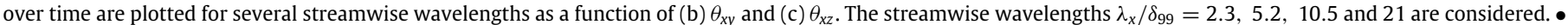
are associated with $\max _{(\beta, t)} \sigma^{2}$ for each $\lambda_{x} / \delta_{99}$. TBL1 is considered for (a), (b) and (c).
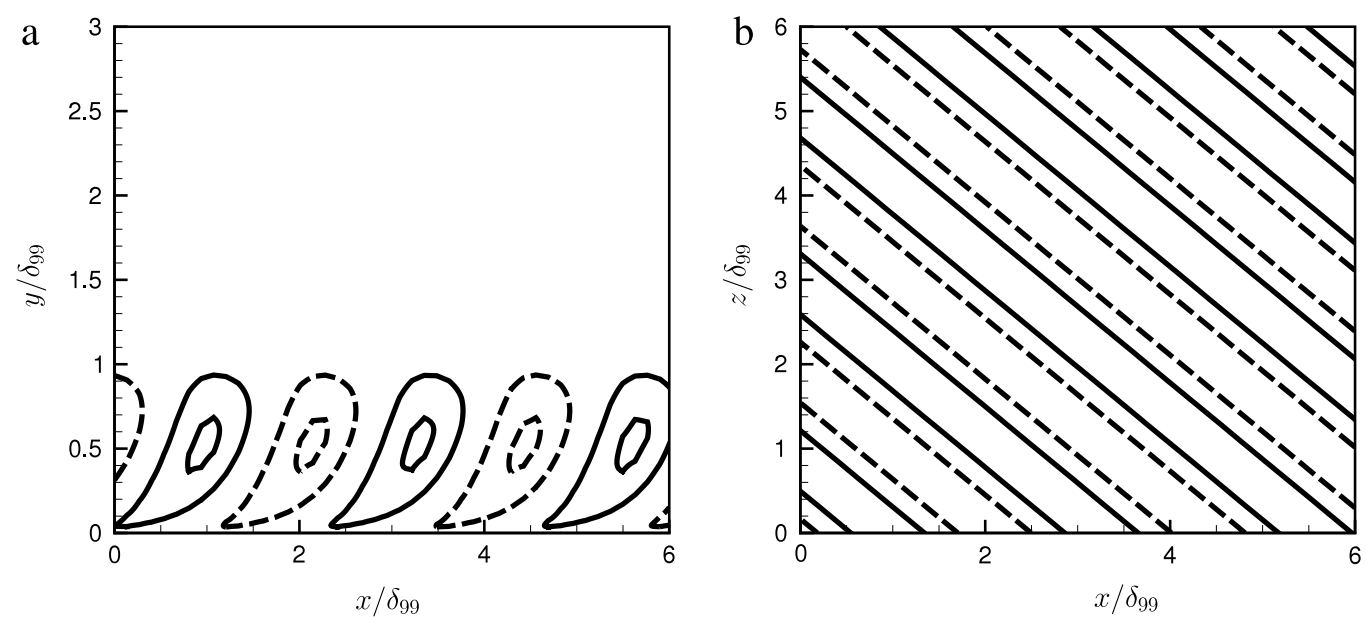

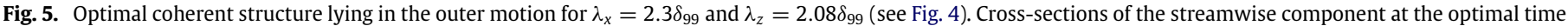
are shown in the plane $(x, y)(\mathrm{a})$ and in the plane $(x, z)$ at $y=0.5 \delta_{99}$ (b). Solid lines represent positive values and dashed lines negative values.

it may be observed that its distribution along the normal direction $y$ starts to grow significantly far from the wall and more specifically in the log layer and reaches a maximum value in the wake region. In addition, a very sharp peak and sign change are observed very near the boundary layer edge in comparison to the inner case. It may be assumed, that is a consequence of the sharp change of the optimal mode near the edge of the boundary layer as depicted in Fig. 3(b) and also shown by Cossu et al. [11]. Such a behaviour could be attributed to the choice of the turbulent viscosity. Indeed, as underlined in Section 2, $v_{t}(y)$ is extrapolated in the potential region by using their computed values inside the boundary layer. Hence, in order to ensure that the next results will be not dependent on the assumptions made in the eddy viscosity, we also report in Fig. 7 the sensitivity functions for the outer peak 

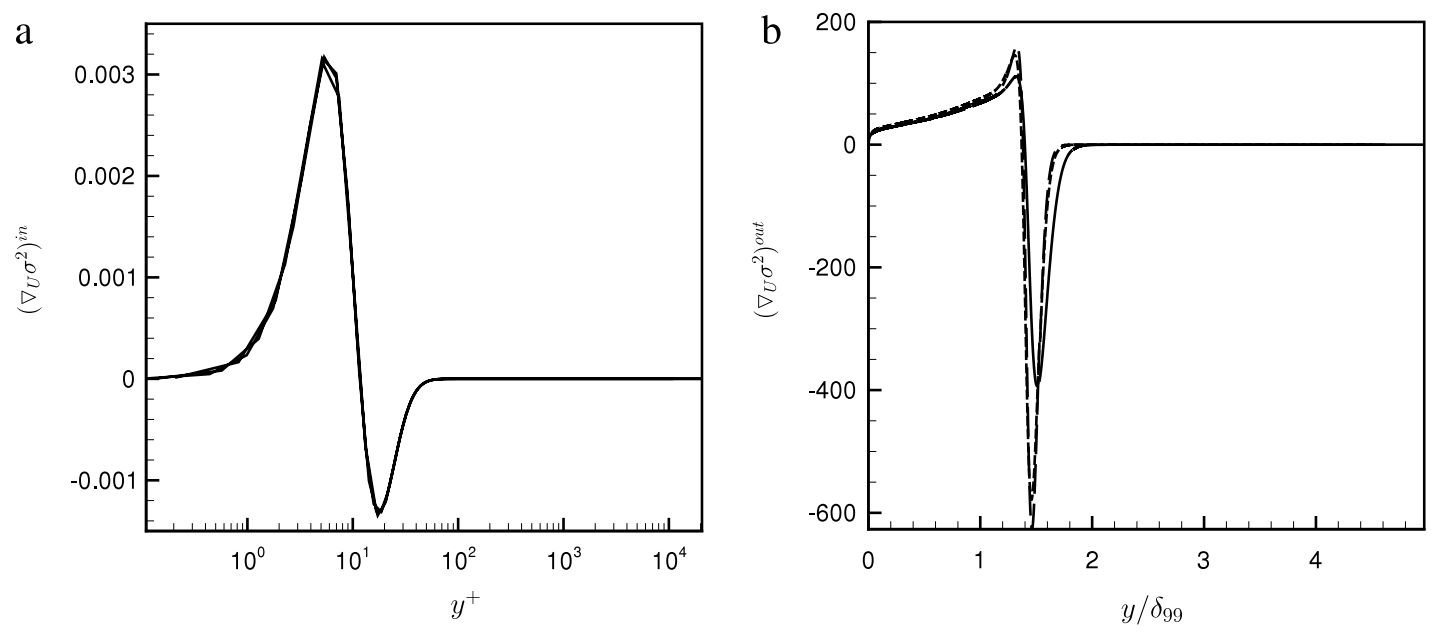

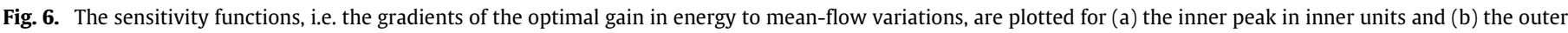
peak in outer units for $v_{t}(y) .-$, TBL1; --- , TBL2; --- , TBL3.

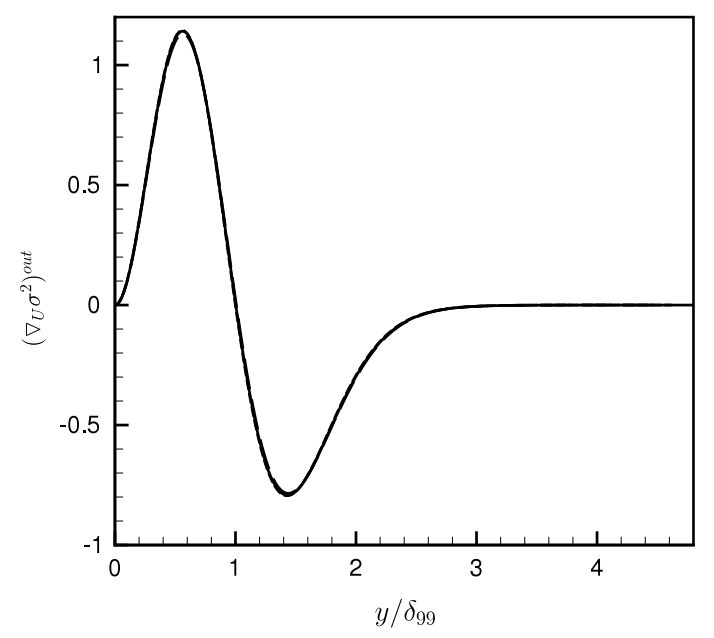

Fig. 7. The sensitivity functions are plotted for the outer peak in outer units for $v_{t}=v_{t \max } \cdot-$, TBL1;,--- TBL2; --- , TBL3.

when $v_{t}$ is assumed to be equal to $v_{t \max }$. In this case, the profiles agree remarkably well with those obtained for the inner peak.

It is worth noting that for the inner peak, the curves corresponding to the optimal mode localized in the inner region collapse in a single curve for all Reynolds numbers when the sensitivity functions are scaled in inner units. The same observation is made for the sensitivity functions of the optimal mode associated with the outer region when using a scaling in outer variables for both the variable eddy viscosity $v_{t}(y)$ and when assuming $v_{t}(y)=v_{t \max }$. In particular, one may observe that the amplitude of the sensitivity function for both the inner and outer peaks is not affected for the range of Reynolds numbers which are considered here. This result is expected for the inner peak by taking into account the fact that the optimal gain is independent of the Reynolds number. On the contrary, as discussed in Section 5.1, the optimal gain in energy for the outer region is dependent on the Reynolds number; the self-similarity for the sensitivity function scaled in outer units is thus not obvious. In Fig. 8, the sensitivity functions for the inner and outer peaks, normalized by the maximum value of their modulus, are superimposed and are displayed in inner (Fig. 8(a), (c)) and outer (Fig. 8(b), (d)) variables. We thus point out that the separation between the spatial support for the sensitivity functions associated with the inner and outer peaks is increasing with the Reynolds number. It gives significant evidence that the mutual dependency between the optimal mode associated with the inner region and the one associated with the outer region is decreasing with an increase in $R e_{\tau}$ (we recall that $R e_{\tau}$ characterizes the scale separation between these regions). Furthermore, this strong separation is a robust feature, not sensitive to the shape of the eddy viscosity, especially at the interface between the boundary layer and the potential region.

Furthermore, as discussed in the previous section, the experiments show that the very large coherent structures which populate the outer motion have a finite size in the streamwise direction. We now investigate the evolution of the sensitivity functions associated with the optimal modes in the outer region for several streamwise wavelengths $\lambda_{x}$. We consider the case TBL1 and the variable eddy viscosity, $v_{t}(y)$, is used. In Fig. 9, sensitivity functions associated with the maximum gain in energy over $\beta$ and $t\left(\max _{(t, \beta)} \sigma^{2}\right)$ for $\lambda_{x}=5.2 \delta_{99}$ and $\lambda_{x}=2.3 \delta_{99}$ are plotted. A clear separation between the distributions of the sensitivity functions for the coherent structures in the outer region having a finite size along $x$ and the one for the inner peak is observed in Fig. 9(a). As for the case $\alpha=0$, this scale separation is expected to increase with $R e_{\tau}$. The evolution of the amplitudes of the sensitivity functions with a decrease of $\lambda_{x}$ is shown in Fig. 9(b). The amplitude of such sensitivity functions is seen to decrease with a decrease in $\lambda_{x}$ and more particularly closed to the overlap region with the sensitivity function for the inner peak. In addition, the sensitivity functions displayed in Fig. 9(b) exhibit a smooth profile in comparison to the sensitivity function associated with the outer peak. It may be the consequence of its spatial support which is drifted towards the wall, behind the edge of the boundary layer. Hence, it may be assumed that the choice of $v_{t}$ has a little influence on the properties of the sensitivity functions associated with finite values of $\lambda_{x}$.

Therefore, we suggest that an optimal base flow deviation aiming to manipulate the coherent structures scaled in inner variables should have a slight impact on the coherent motion of the outer region. In addition, this impact is expected to decrease with the increase of $R e_{\tau}$.

\subsection{Optimal deviation for the optimal mode in the inner region and its influence on the outer region}

The optimal mode associated with the inner layer (inner peak) is selected and the optimal distorted mean flow is tracked until a residual (with respect to variations in $\sigma^{2}$ between two successive iterations) drops below a threshold value fixed to $10^{-10}$. We recall that a stabilizing effect is investigated (see Eq. (19)). We wish to minimize the singular value which has the largest magnitude. Hence, we need to ensure that our optimization does not affect the 

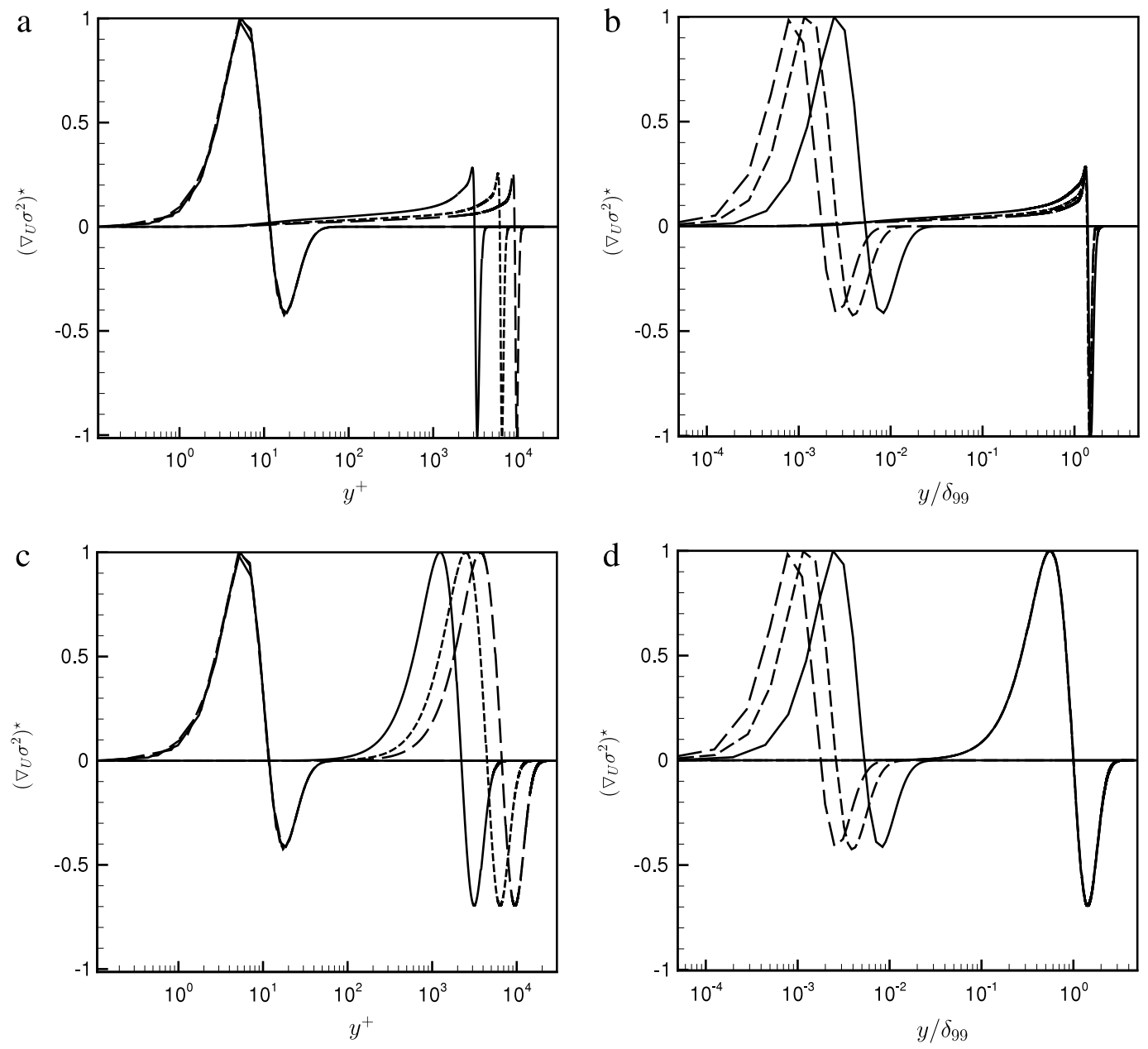

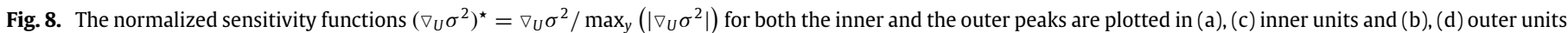
for $v_{t}(y)((\mathrm{a})$ and $(\mathrm{b}))$ and $v_{t \max }((\mathrm{c})$ and $(\mathrm{d})) .-$, TBL1;,--- TBL2; - - -, TBL3.
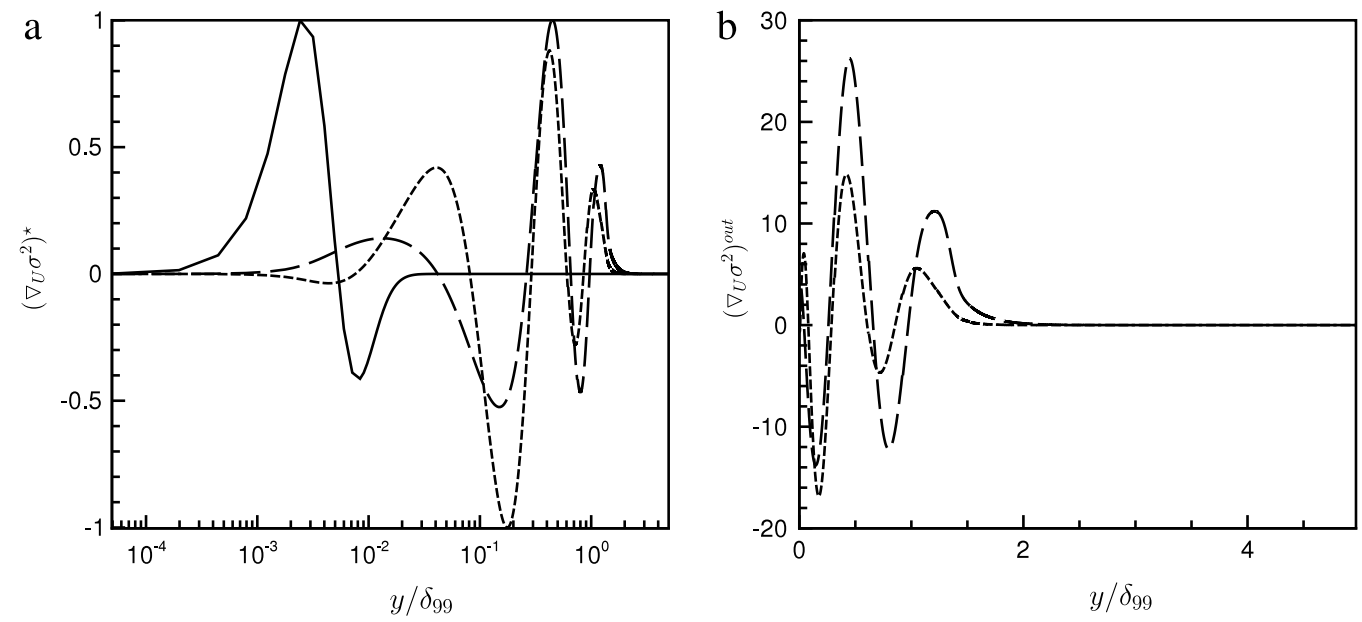

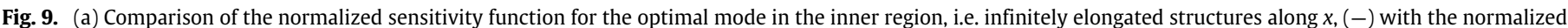

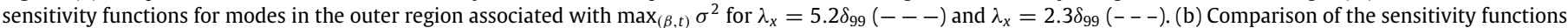
for modes in the outer region associated with $\max _{(\beta, t)} \sigma^{2}$ for $\lambda_{x}=5.2 \delta_{99}(---), 2.3 \delta_{99}(---)$. The case TBL1 is considered.

least damped singular values. To elucidate this specific point, the singular value decomposition is provided in Fig. 10 where the inner peak, corresponding to the couple $\left(\lambda_{z}^{+}, t^{+}\right)=(81,15)$, is targeted for TBL1. One may notice that only one singular value is amplified for $r=0$ (i.e. the mean flow is not modified). In addition, this result still holds when $r$ is increasing.
Let us consider the optimal mean flow deviation $U$ associated with the inner peak for a given value of $r$. The parameters space $\left(\lambda_{z}=2 \pi / \beta, t\right)$ is rescanned in order to check that a stabilizing mean flow distortion for the optimal gain at $r=0\left(\lambda_{z}^{+}=81\right.$ and $\left.t^{+}=15\right)$ does not produce an increase in $\sigma^{2}$ for a different couple $\left(\lambda_{z}^{+}, t^{+}\right)$. In Fig. 11, we show the evolution of the singular value $\sigma^{2}$ 


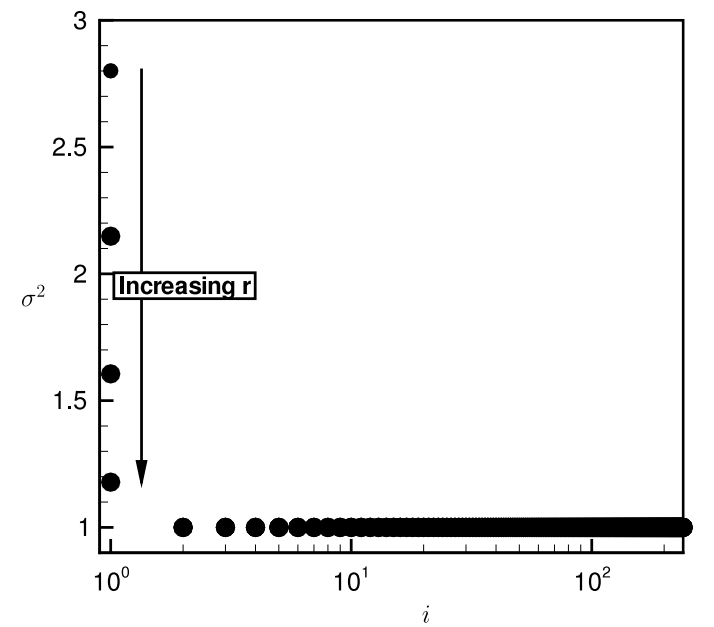

Fig. 10. The singular values for $\left(\lambda_{z}^{+}, t^{+}\right)=(81,15)$ are plotted for $r=0, r=$ $0.005, r=0.01$ and $r=0.015$ for TBL1.

computed by using $U$ as the new mean flow for several $\lambda_{z}^{+}$and $t^{+}$. Two values of $r$ are considered and the turbulent boundary layer TBL1 is chosen as an example. It is thus shown that we are able to stabilize the inner peak as well as a wide range of $\alpha^{+}$and $t^{+}$ close to the maximum amplification of the coherent structures in the inner region. The latter figure also shows a shift of the inner peak to the outer region when $r$ is increasing, which has for consequences to increase its characteristic spanwise wavelength and its characteristic time scale. Finally, the double peak structure for $\sigma^{2}$ in the plane $\left(\lambda_{z}^{+}, t^{+}\right)$is almost vanishing for $r=0.015$. On the opposite, the influence of such an optimal deviation is negligible in the neighbourhood of the outer peak. It confirms the discussion of Section 5.2 where a large separation in terms of spatial support for the sensitivity functions with respect to coherent structures evolving in inner and outer regions is observed. In Fig. 12, the evolution of $\sigma^{2}$ with $r$ for TBL1, 2 and 3 is depicted for the inner peak. When the Reynolds number is increased, the magnitude of the mean flow deviation which is needed to stabilize the optimal gain is lower. Such a behaviour could not be caused by the amplitude of the sensitivity function which is not dependent on the Reynolds number (see Fig. 6(a)). However, as discussed above, the separation between the inner and outer regions in terms of sensitivity functions is increasing with the Reynolds number $R e_{\tau}$. Hence, it may be argued that such an increase in terms of scale separation

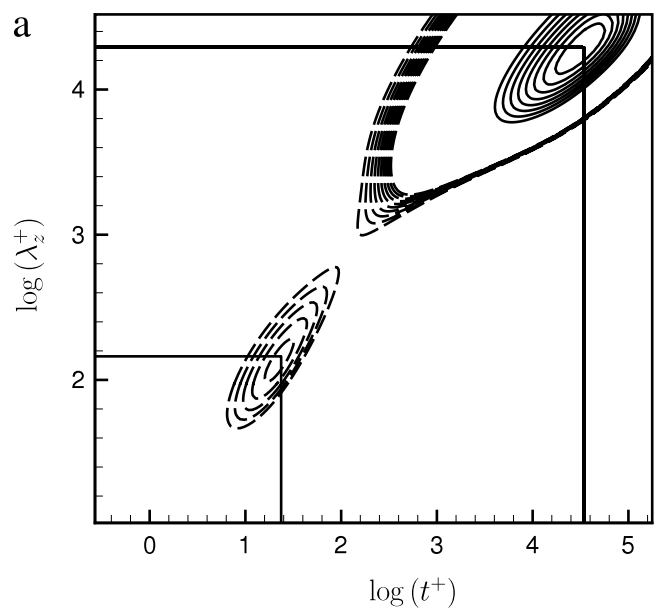

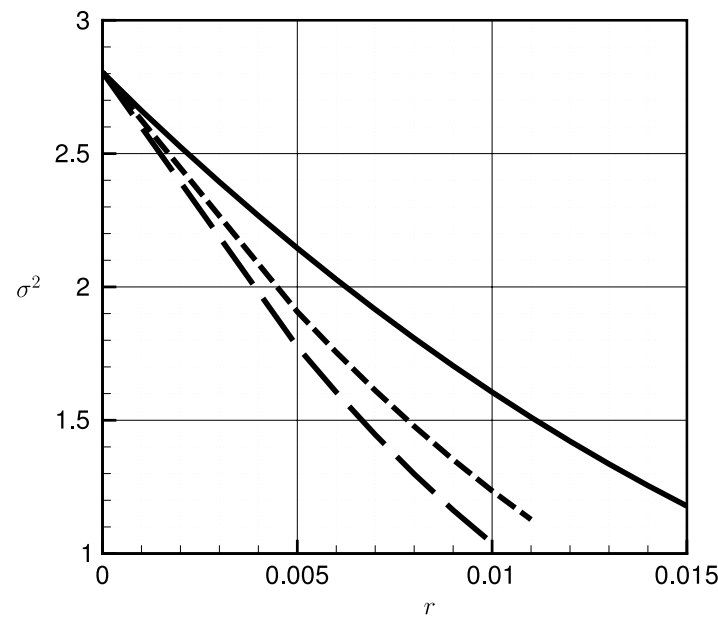

Fig. 12. The evolution of the leading singular value $\sigma^{2}$ for $\lambda_{z}^{+}=81$ and $t^{+}=15$ (inner peak) is plotted along $r$. - , TBL1; - - , TBL2; --- , TBL3.

leads to a more efficient control to stabilize the inner peak. To further support this hypothesis, the optimal gain deviation for the parameters space $\left(\lambda_{z}^{+}, t^{+}\right)$is investigated for TBL2 and TBL3. We fix $r$ equal to 0.011 and 0.0095 for TBL2 and TBL3 in order to compare the case $r=0.015$ for TBL1. Indeed, by considering such values for TBL1, TBL2 and TBL3, a similar optimal gain deviation is obtained (see Fig. 12). In Figs. 13 and 14, we show the evolution of $\sigma^{2}$ for various $\left(\lambda_{z}^{+}, t^{+}\right)$associated with the previous values of $r$ for TBL2 and TBL3. One may observe a similar behaviour as the one described for TBL1. In particular, the inner peak is shifted for a larger value of both $\lambda_{z}^{+}$and $t^{+}$and almost vanishes. It is also interesting to notice that the maximum gain, for the deviation parameters considered, is reached for the same couple $\left(\lambda^{+}, t^{+}\right)$and has an almost equal value for all the Reynolds numbers. We also observe that as the Reynolds number is increased, a clear distinction appears between the optimal gain deviation for the inner peak and outer peak. It is thus consistent with the above discussion about the evolution of the scale separation between the inner and outer regions with $R e_{\tau}$.

The turbulent mean flow modification for $r$ varying from 0 to 0.015 is displayed in Fig. 15 for TBL1. One may observe that the mean flow deviation is mainly localized in the inner wall region and buffer layer, which is in agreement with the sensitivity function shown in Fig. 6. Let us now examine the influence of a small deviation in a turbulent mean flow profile on the eigenfunctions. The case TBL1 is considered as an example. We

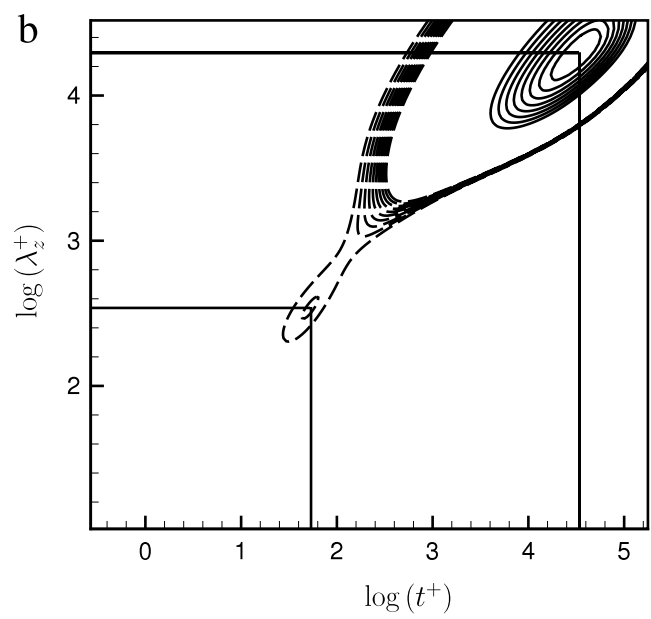

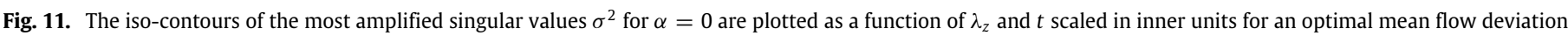

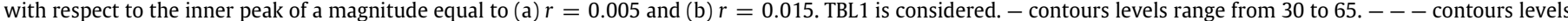
range from 1.8 to 2.7 . 

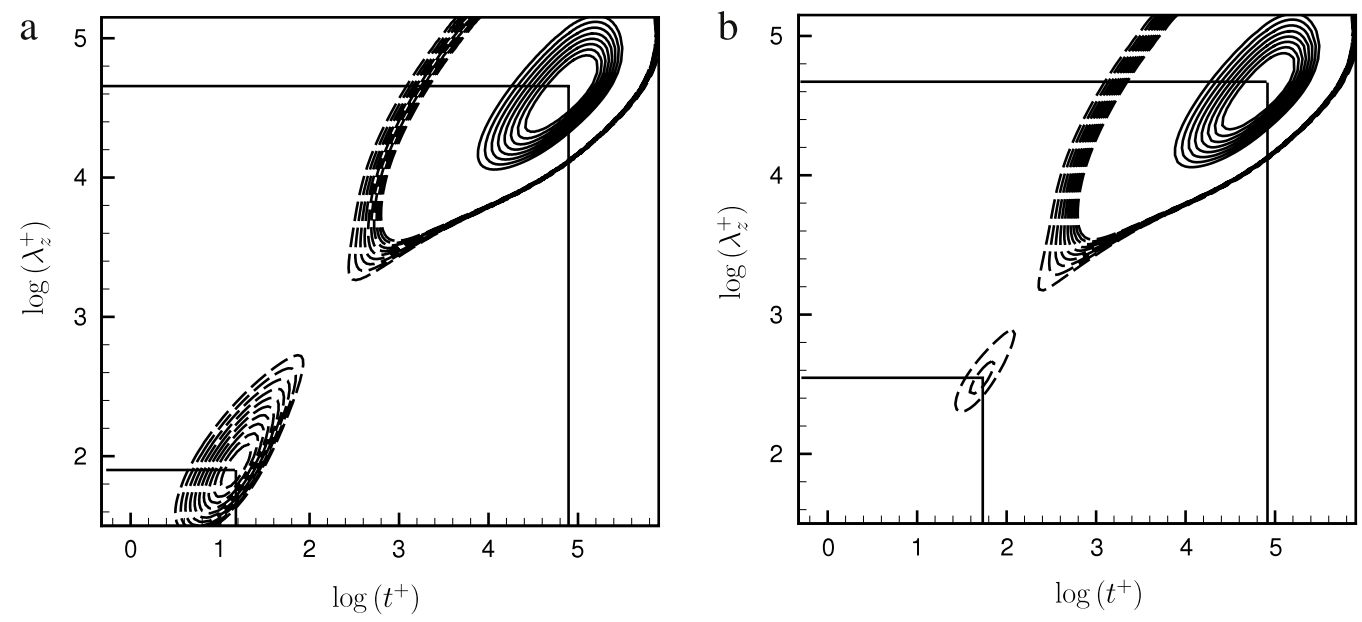

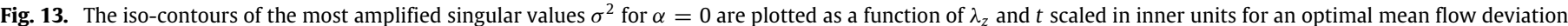

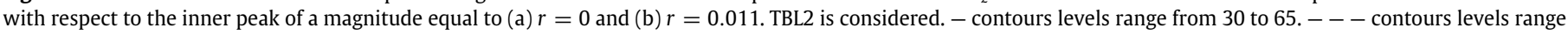
from 1.8 to 2.7 .
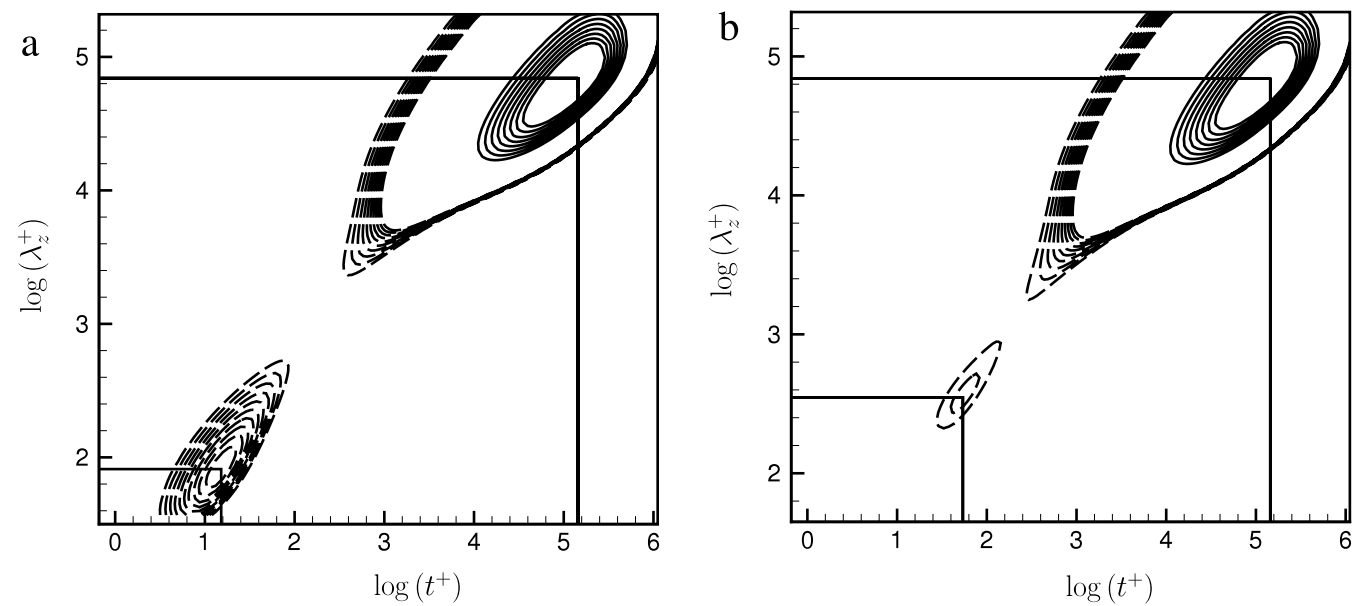

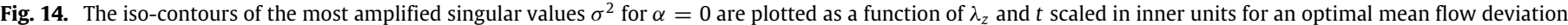

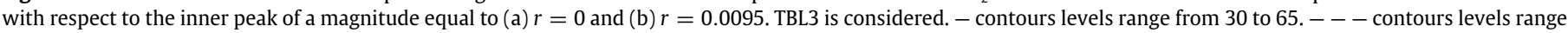
from 1.8 to 2.7 .

first investigate the evolution of the eigenfunction for the inner peak with respect to $r$ as displayed in Fig. 11. The eigenfunctions for $r=0, r=0.005$ and $r=0.015\left(\left(\lambda_{z}^{+}, t^{+}\right)=(81,15)\right.$, $(147,23)$ and $(342,52)$ respectively, see Figs. 2(a) and 11(a), (b)), are depicted in Fig. 16. One may observe that for both cases the initial condition is dominated by the normal and spanwise (not shown here) velocity components whereas at the optimal time $\left(t_{\mathrm{opt}}\right)$ the coherent motion is governed by the streamwise velocity component. Such a similarity suggests that a lift-up mechanism is responsible for the transient energy growth for all deviation parameters which are considered. However, it also illustrates that the spatial extension for the optimal mode is increasing with $r$, until to reach large values in the logarithmic layer for $r=0.015$. This observation is consistent with the drift of the inner peak to the outer region as discussed above. Now, we consider the evolution of eigenfunctions associated with the outer peak when the inner peak is targeted. One may observe in Fig. 17 that the different curves for $r$ varying from 0 to 0.015 are collapsing.

Finally, the influence of the optimal mean flow deviation, as shown in Fig. 15, onto the outer motion is investigated for $\alpha \neq$ 0 . For TBL1, the same contours displayed in Fig. 4 are shown in Fig. 18 for the deviation parameter $r=0.015$. An almost perfect collapsing is observed in Fig. 18 giving strong evidence about Townsend's wall similarity hypothesis [17]. A similar behaviour

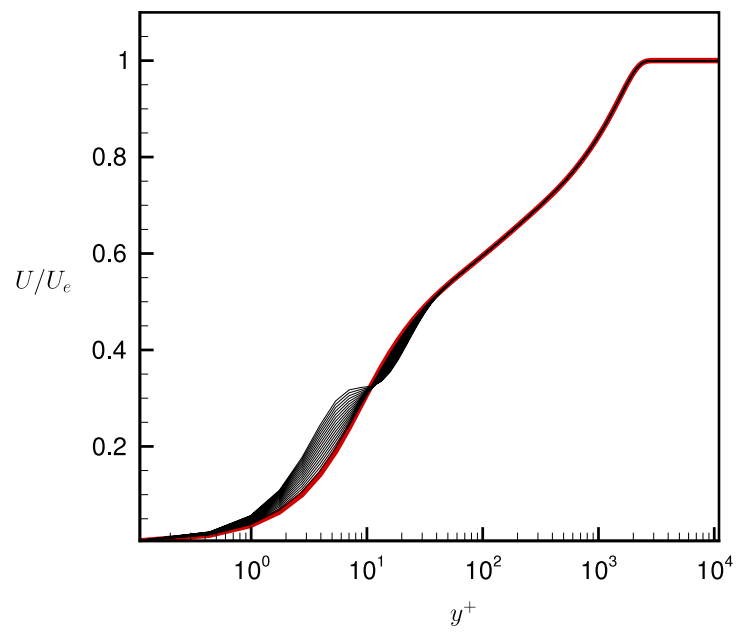

Fig. 15. The mean flow modification for $r$ varying from $r=0$ (in red) and $r=0.015$ is plotted for TBL1. (For interpretation of the references to colour in this figure legend, the reader is referred to the web version of this article.)

for TBL2 and TBL3 is expected by considering that the scale separation between the inner and outer regions is increasing with 

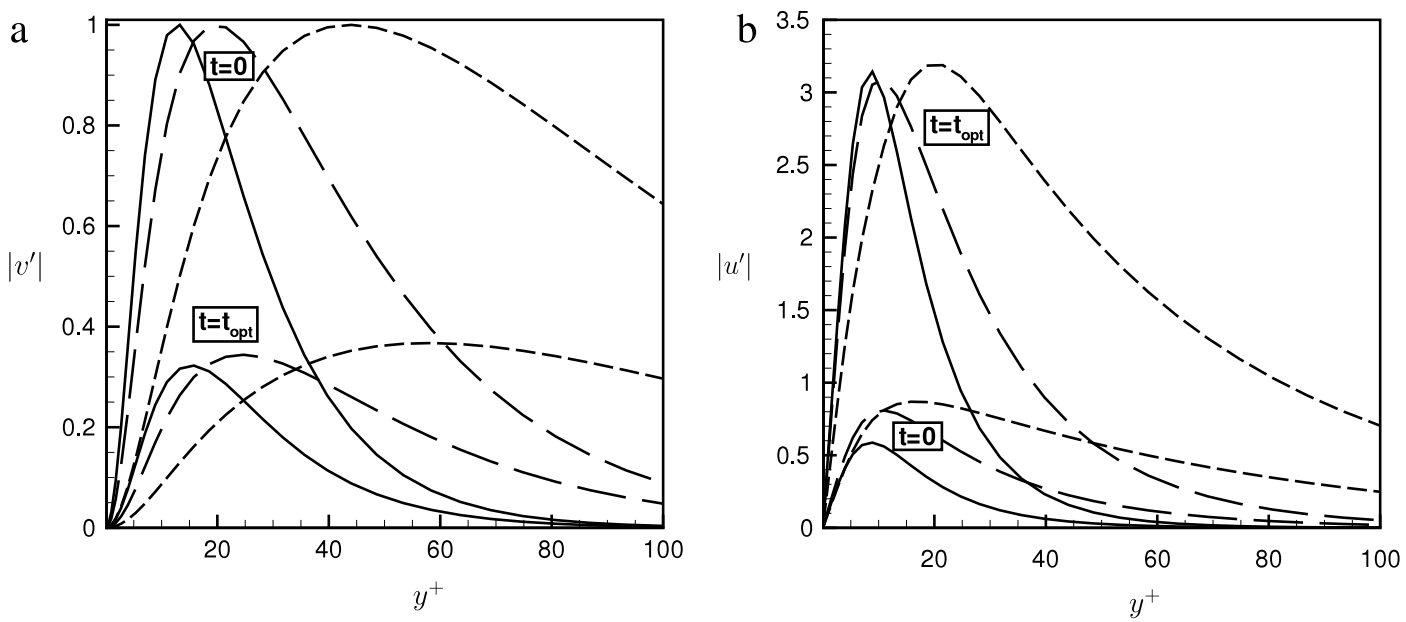

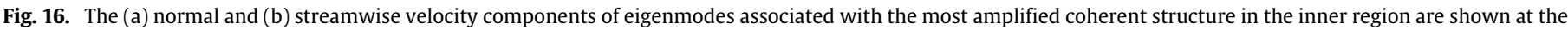
initial time and the optimal time for TBL1.,$- r=0 ;---, r=0.005 ;---, r=0.015$.
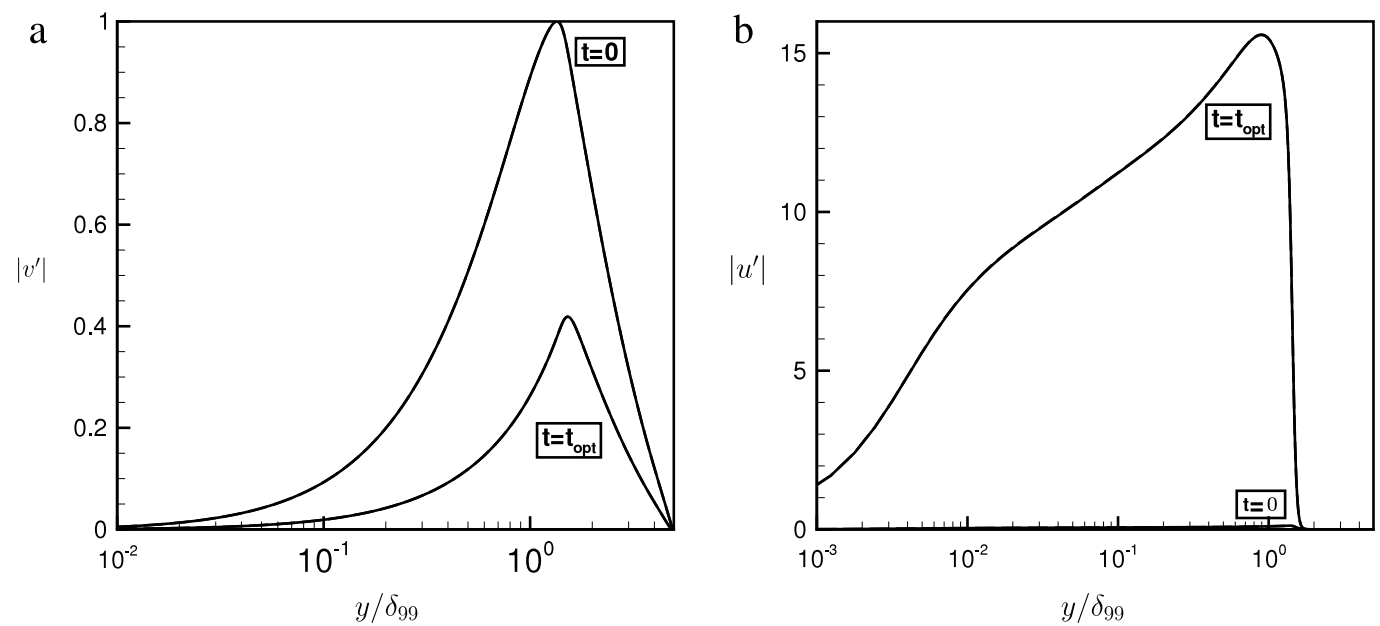

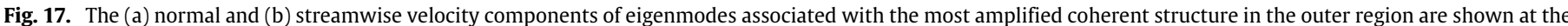
initial time and the optimal time for TBL1.,$- r=0 ;---, r=0.005 ;---, r=0.015$.

$R e_{\tau}$. Furthermore, the position of $\max _{(\beta, t)} \sigma^{2}$ is unaffected by the mean flow modification.

\section{Discussion}

As recently proved by Hwang and Cossu [16] in their Large Eddy Simulations of a turbulent channel flow, the large-scale motion in the outer layer may not rely on the existence of smaller scale structures. In particular, the authors suggest that the streaky structures which populate the outer region may experience an autonomous cycle, similar to the self-sustaining process observed in the inner and buffer layer. Hwang and Cossu [16] conjecture thus that such a self-sustained mechanism which occurs in the outer motion for a turbulent channel flow exists for other turbulent canonical shear flows. They also observe that the very large-scale motion is not necessarily the result from the concatenation of streaks at smaller scales (the so-called very large-scale model [14]). By assuming that the singular value decomposition of the governing operator developed by Reynolds and Hussain [6] is able to describe the linear amplification of coherent structures in turbulent mean flows, the present study further supports such a way of thinking. In particular, we show that very large structures scaled in outer units may be amplified in a turbulent boundary layer while the near-wall cycle is not active, i.e. damped by a small near-wall mean flow modification. This result is clearly highlighted through optimizations aiming to damp the inner layer structures. The optimal mean flow modification is thus seen to not modify the amplification of very large-scale motion in the outer region. Such a fact gives strong evidence about Townsend's hypothesis [17] which has been widely studied both in experiments and in numerical simulations for turbulent boundary layers with rough walls.

Furthermore, researchers agree that the events associated with the self-sustained process in the near-wall are strongly driving both the production of the Reynolds stress and kinetic energy [3]. In this context, the linear amplification of streaky structures plays an important part in such a process. Hence, in the light of the influence of small mean flow deviation on the attenuation of optimal streaks scaled in inner units, one may discuss about the control of nearwall motion. For that purpose, we may compare our theoretical results with the direct numerical simulation of a turbulent channel flow forced by a steady streamwise forcing localized close to the wall by $\mathrm{Xu}$ et al. [34]. The latter authors illustrate that the imposition of a specific streamwise steady forcing modifies both the mean flow and turbulence near the wall. In particular, $\mathrm{Xu}$ et al. [34] use a steady forcing which decelerates the flow close to the wall and then accelerates the flow in the adjacent region within a distance of 20 wall units. For a small amplitude, the forcing leads to the formation of a shear layer close to the wall and as for consequence to both reduce the Reynolds shear stress and the skin friction. In addition, it is observed by $\mathrm{Xu}$ et al. [34] 

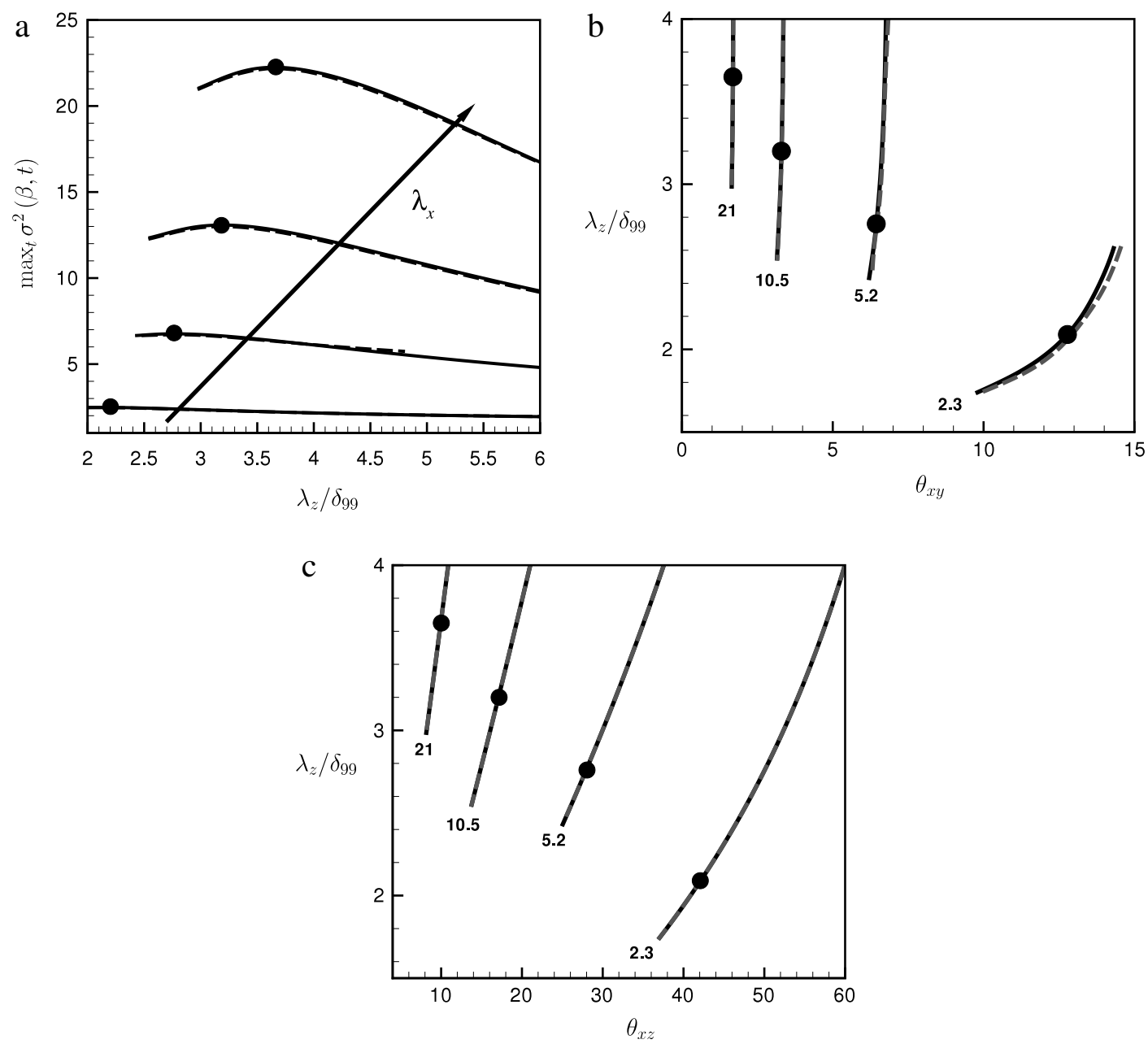

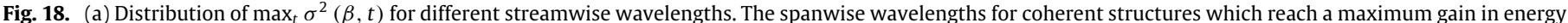

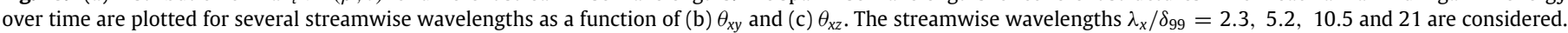

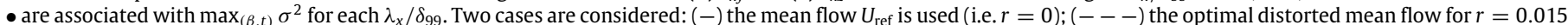
with respect to the inner peak is used. The case TBL1 is considered.

that the usual self-sustained process in the near-wall is reduced and replaced by the structures associated with the shear layer. One may suppose that this mechanism can be extended to various turbulent shear flows, such as the turbulent boundary layer. Hence, the study of Xu et al. [34] exhibits a control mechanism which closely resembles the one derived from our optimal mean flow deviation, as depicted in Fig. 15, aiming to damp the optimal mode associated with streaks' amplification in the inner region.

\section{Conclusion}

In conclusion, the present study extends the sensitivity analysis based on optimal transient growth developed in a laminar framework by Brandt et al. [28] to turbulent flows. The existence of two overlapping regions is a common feature of turbulent shear flows, such as pipes, channels, and boundary layers. In that respect, the case of the turbulent boundary layer is chosen, which may be considered as a canonical turbulent shear flow. It is illustrated, through a sensitivity analysis of mean flow deviation, that the amplification processes of coherent structures which populate the inner and outer regions are decoupled. In addition, our analysis could provide an efficient tool to design the control strategy for turbulent flows by acting on coherent structures. Finally, the extension of our study to nonparallel turbulent flows, for instance separated flows, could be an interesting prospect.

\section{Acknowledgements}

The authors are grateful to Prof. Patrick Huerre for these courses and many works that have inspired several generations of students and researchers.

\section{Appendix. Adjoint operator $\mathcal{A}^{+}$}

The derivation of the adjoint operator of $\mathcal{A}$ associated with the inner product $\langle$,$\rangle is found by using an analytical expression of$ $\left\langle\hat{\mathbf{u}}^{+}, \mathcal{A} \hat{\mathbf{u}}\right\rangle$. The latter expression is rewritten as

$$
\begin{aligned}
& \int_{0}^{y_{\max }}\left\{i \alpha \hat{u} U+\hat{v} \frac{d U}{d y}+\left(\frac{1+v_{t}}{\operatorname{Re}}\right)\left(\alpha^{2}+\beta^{2}\right) \hat{u}\right. \\
& \left.-\frac{\partial}{\partial y}\left(\frac{1+v_{t}}{\operatorname{Re}} \frac{\partial \hat{u}}{\partial y}\right)-i \alpha \frac{d}{d y}\left(\frac{v_{t}}{\operatorname{Re}}\right) \hat{v}+i \alpha \hat{p}\right\}\left(\hat{u}^{+}\right)^{*} \\
& \quad \times\left\{i \alpha \hat{v} U+\left(\frac{1+v_{t}}{\operatorname{Re}}\right)\left(\alpha^{2}+\beta^{2}\right) \hat{v}-\left(\frac{1+v_{t}}{\operatorname{Re}}\right) i \alpha \frac{\partial \hat{u}}{\partial y}\right. \\
& \left.-\frac{\partial}{\partial y}\left(\frac{1+v_{t}}{\operatorname{Re}} 2 \frac{\partial \hat{v}}{\partial y}\right)-\left(\frac{1+v_{t}}{\operatorname{Re}}\right) i \beta \frac{\partial \hat{w}}{\partial y}+\frac{\partial \hat{p}}{\partial y}\right\}\left(\hat{v}^{+}\right)^{*} \\
& +\left\{i \alpha \hat{w} U+\left(\frac{1+v_{t}}{\operatorname{Re}}\right)\left(\alpha^{2}+\beta^{2}\right) \hat{w}-\frac{d}{d y}\left(\frac{v_{t}}{\operatorname{Re}}\right) i \beta \hat{v}\right. \\
& \left.-\frac{\partial}{\partial y}\left(\frac{1+v_{t}}{\operatorname{Re}} \frac{\partial \hat{w}}{\partial y}\right)+i \beta \hat{p}\right\}\left(\hat{w}^{+}\right)^{*} d y .
\end{aligned}
$$


By using $i \alpha \hat{u}+i \beta \hat{w}+d \hat{v} / d y=0$, we recover the expression of the operator $\mathcal{A}$ given in Section 3.

After integration by parts, the previous equation (A.1) leads to

$$
\begin{aligned}
& \int_{0}^{y_{\max }}\left\{-i \alpha U \hat{u}^{+}+\left(\frac{1+v_{t}}{\operatorname{Re}}\right)\left(\alpha^{2}+\beta^{2}\right) \hat{u}^{+}\right. \\
& -\left(\frac{1+v_{t}}{\operatorname{Re}}\right) \frac{\partial^{2} \hat{u}^{+}}{\partial y^{2}}-\frac{d}{d y}\left(\frac{v_{t}}{\operatorname{Re}}\right) \frac{\partial \hat{u}^{+}}{\partial y}-i \alpha \frac{1+v_{t}}{\operatorname{Re} \frac{\partial \hat{v}^{+}}{\partial y}} \\
& \left.-\frac{d}{d y}\left(\frac{v_{t}}{\operatorname{Re}}\right) i \alpha \hat{v}^{+}+i \alpha p^{+}\right\}^{*} \hat{u} \\
& +\left\{-i \alpha U \hat{v}^{+}-\frac{d U}{d y} \hat{u}^{+}+\left(\frac{1+v_{t}}{\operatorname{Re}}\right)\left(\alpha^{2}+\beta^{2}\right) \hat{v}^{+}\right. \\
& \left.-3 \frac{d}{d y}\left(\frac{v_{t}}{\operatorname{Re}}\right) \frac{\partial \hat{v}^{+}}{\partial y}-2 \frac{1+v_{t}}{\operatorname{Re}} \frac{\partial^{2} \hat{v}^{+}}{\partial y^{2}}+\frac{\partial \hat{p}^{+}}{\partial y}\right\}^{*} \hat{v} \\
& +\left\{-i \alpha U \hat{w}^{+}+\left(\frac{1+v_{t}}{\operatorname{Re}}\right)\left(\alpha^{2}+\beta^{2}\right) \hat{w}^{+}-\frac{1+v_{t}}{\operatorname{Re}} i \frac{\partial \hat{v}^{+}}{\partial y}\right. \\
& -i \beta \frac{d}{d y}\left(\frac{v_{t}}{\operatorname{Re}}\right) \hat{v}^{+}-\frac{1+v_{t}}{\operatorname{Re}} \frac{\partial^{2} \hat{w}^{+}}{\partial y^{2}} \\
& \left.-\frac{d}{d y}\left(\frac{v_{t}}{\operatorname{Re}}\right) \frac{\partial \hat{w}^{+}}{\partial y}+i \beta \hat{p}^{+}\right\}^{*} \hat{w} d y .
\end{aligned}
$$

\section{References}

[1] S.J. Kline, W.C. Reynolds, F.A. Schraub, P.W. Runstadler, Structure of turbulent boundary layers, J. Fluid Mech. 30 (1967) 741-773.

[2] J. Jimenez, Near-wall turbulence, Phys. Fluids 25 (2013) 101302.

[3] R.L. Panton, Overview of the self-sustaining mechanisms of wall turbulence, Prog. Aerosp. Sci. 37 (2001) 341-383.

[4] J.M. Hamilton, J. Kim, F. Waleffe, Regeneration mechanisms of near-wall turbulence structures, J. Fluid Mech. 287 (1995) 317-349.

[5] J. Jimenez, How linear is wall-bounded turbulence, Phys. Fluids 25 (2013) 110814.

[6] W.C. Reynolds, K.M.F. Hussain, The mechanics of an organized wave in turbulence shear flow. Part 3. Theoretical models and comparisons with experiments, J. Fluid Mech. 52 (1972) 263-288.

[7] J.C. Del Alamo, J. Jimenez, P. Zandonade, R.D. Moser, Scaling of the energy spectra of turbulent channels, J. Fluid Mech. 500 (2004) 135-144.

[8] K.M. Butler, B.F. Farrel, Optimal perturbations and streak spacing in wallbounded turbulent shear flow, Phys. Fluids 5 (1992) 774-777.

[9] P.J. Schmid, D.S. Henningson, Stability and Transition in Shear Flows, Springer, 2001.
[10] G. Pujals, M. Garci-Villalba, C. Cossu, S. Depardon, A note on optimal transient growth in turbulent channels flows, Phys. Fluids 21 (2009) 01519.

[11] C. Cossu, G. Pujals, S. Depardon, Optimal transient growth and very large-scale structures in turbulent boundary layers, J. Fluid Mech. 619 (2009) 79-94.

[12] N. Hutchins, I. Marusic, Evidence of very long meandering features in the logarithmic region of turbulent boundary layers, J. Fluid Mech. 579 (2007) $1-28$.

[13] A.S. Sharma, B.J. McKeon, On coherent structure in wall turbulence, J. Fluid Mech. 728 (2013) 196-238.

[14] K.C. Kim, R.J. Adrian, Very large-scale motion in the outer layer, Phys. Fluids 11 (1999) 417-422.

[15] Y. Mizuno, J. Jimenez, Wall turbulence without walls, J. Fluid Mech. 723 (2013) 429-455.

[16] Y. Hwang, C. Cossu, Self-sustained process at large scales in turbulent channe flow, Phys. Rev. Lett. 105 (2010) 044505.

[17] A.A. Townsend, The Structure of Turbulent Shear Flow, University of Cambridge, 1976.

[18] K.A. Flack, M.P. Schultz, T.A. Shapiro, Experimental support for Townsend's Reynolds number similarity hypothesis on rough walls, Phys. Fluids 17 (2005) 035102.

[19] M.A. Amir, I.P. Castro, Turbulence in rough-wall boundary layers: universality issues, Exp. Fluids 51 (2011) 313-326.

[20] P.A. Krogstad, V. Efros, About turbulence statistics in the outer part of a boundary layer developing over two-dimensional surface roughness, Phys. Fluids 24 (2012) 075112.

[21] R.J. Volino, M.P. Schultz, K.A. Flack, Turbulence structure in a boundary layer with two-dimensional roughness, J. Fluid Mech. 635 (2009) 75-101.

[22] O. Flores, J. Jimenez, Effect of wall-boundary disturbances on turbulent channel flows, J. Fluid Mech. 566 (2006) 357-376.

[23] S.H. Lee, H.J. Sung, Direct numerical simulation of the turbulent boundary layer over a rod-roughened wall, J. Fluid Mech. 584 (2007) 125-146.

[24] J. Jimenez, Turbulent flows over rough walls, Annu. Rev. Fluid Mech. 36 (2004) 173-196.

[25] A. Bottaro, P. Corbett, P. Luchini, The effet of base flow variation on flow stability, J. Fluid Mech. 476 (2003) 293-302.

[26] O. Marquet, D. Sipp, L. Jacquin, Sensitivity analysis and passive control of cylinder flow, J. Fluid Mech. 615 (2008) 221-252.

[27] P. Meliga, G. Pujals, E. Serre, Sensitivity of 2D turbulent flow past a D-shaped cylinder using global stability, Phys. Fluids 24 (2012) 061701.

[28] L. Brandt, D. Sipp, J. Pralits, O. Marquet, Effect of base-flow variation in noise amplifiers: the flat plate boundary layer, J. Fluid Mech. 687 (2011) 503-528.

29] P.A. Monkewitz, K.A. Chauhan, H.M. Nagib, Self-consistent high-Reynoldsnumber asymptotics for zero-pressure-gradient turbulent boundary layers, Phys. Fluids 19 (2007) 115101

[30] R.B. Sidje, Expokit: a software package for computing matrix exponentials, ACM Trans. Math. Software 24 (1998) 130-156.

[31] C.D. Tomkins, R.J. Adrian, Spanwise structure and scale growth in turbulent boundary layers, J. Fluid Mech. 490 (2003) 37-74.

[32] I. Marusic, W.D.C. Heuer, Reynolds number invariance of the structure inclination angle in wall turbulence, Phys. Rev. Lett. 99 (2007) 114504.

[33] K.S. Choi, Near-wall structure of a turbulent boundary layer with riblets, J. Fluid Mech. 208 (1989) 417-458.

[34] J. Xu, S. Dong, M.R. Maxey, G.E. Karniadakis, Turbulent drag reduction by constant near-wall forcing, J. Fluid Mech. 582 (2007) 79-101. 